\title{
FREMDSPRACHERWERB BEI KINDERN MIT LERNSCHWIERIGKEITEN: EINE FALLSTUDIE
}

\author{
Dunja Hajduković \\ Osnovna škola Franje Krežme Osijek \\ Melita Aleksa Varga* \\ Filozofski fakultet Sveučlišta Josipa Jurja Strossmayera u Osijeku
}

\begin{abstract}
Der vorliegende Beitrag beschreibt die Ergebnisse einer Fallstudie im Zusammenhang mit dem Spracherwerb bei Kindern mit Lernschwierigkeiten. Der Beitrag konzentriert sich auf Lernschwierigkeiten, insbesondere auf Störungen und Ursachen, die das Erlernen von Deutsch als Fremdsprache beeinträchtigen, wie ADHS, Dyslexie, Intelligenzminderung und Sprachentwicklungsstörung. Der Schwerpunkt liegt auf ADHS und dem Unterrichten von Deutsch als Fremdsprache auf der Stufe B1. Die Fallstudie befasst sich mit dem Fall Duma, einem Jungen mit mehreren komorbiden Lernschwierigkeiten. Es werden Methoden und Verfahren beschrieben, welche bei der Planung einer individuell angepassten Unterrichtsstunde helfen können - eine ADHS-Diagnosecheckliste, der MoCA-Test, die Einschätzung kommunikativer Fähigkeiten und ein Lernstil-Test. Diese Methoden und Verfahren können von jedem Lehrer selbst durchgeführt werden.
\end{abstract}

Schlüsselwörter: Spracherwerb, Lernschwierigkeiten, ADHS, Dyslexie, Sprachentwicklungsstörung, Intelligenzminderung, Fallstudie.

\section{EINFÜHRUNG}

Im Rahmen des täglichen Unterricht triffen Lehrer oft auf Kinder mit unterschiedlichen Lernschwierigkeiten. Dabei ist man sehr oft nicht sicher, um welche Lernschwierigkeit, Lernstörung oder Lernschwäche es sich bei dem Kind handelt. Dennoch muss der Lehrer seinen Unterricht so erfolgreich wie möglich durchführen. Im vorliegeden Beitrag werden die Begriffe wie Lernschwierigkeit, Lernbeeinträchtigung, Lernbehinderung, Lernstörung und Lernschwäche genauer erklärt. Es wird auch versucht, unterschiedliche Diagnosemöglichkeiten zu veranschaulichen, mit denen der Lehrer selbst die entsprechende Lernhinderung definieren und folglich auch genaue Maßnahmen unternehmen könnte. Der Beitrag ist Teil einer Pilotstudie ${ }^{1}$, die die erste Autorin dieses Beitrags mit einem Schüler

\footnotetext{
*dhajdukovic93@gmail.com

*** maleksa@ffos.hr

${ }^{1}$ Die Pilotstudie wurde im Rahmen der unveröffentlichten Diplomarbeit „Spracherwerb bei Kindern mit Lernschwierigkeiten“ der Autorin Dunja Hajduković durchgeführt.
} 
durchgeführt hatte, um die entsprechenden Verfahren in der Deutschstunde auswählen zu können. Die Fallstudie befasste sich mit dem Fall von Duma², einem Jungen mit mehreren komorbiden Lernschwierigkeiten. Die Studie wurde mit Einhaltung aller ethischen Richtlinien und relevanten Gesetze bezüglich der Datenschutz-Grundverordnung durchgeführt. Im Beitrag wurden Methoden und Verfahren präsentiert, welche für die Planung einer individuell angepassten Unterrichtsstunde nötig waren - eine ADHSDiagnosecheckliste, der MoCA-Test, die Einschätzung kommunikativer Fähigkeiten und ein Lernstil-Test. Der Zweck dieses Beitrags und des in seinem Rahmen präsentierten Verfahrens ist es, den Deutschlehrern einige Richtlinien $\mathrm{zu}$ bieten, die womöglich im täglichen Unterricht behilflich sein könnten, da wir in der uns zur Verfügung stehenden Literatur keine ähnlichen Studien und Untersuchungen im Bereich Spracherwerb bei komorbiden Lernschwierigkeiten gefunden haben.

\section{BEGRIFFSBESTIMMUNG}

Lernschwierigkeit, Lernbeeinträchtigung, Lernbehinderung, Lernstörung, Lernschwäche sind Begriffe, die im Alltag synonym benutzt werden, aber dennoch Termini für zu unterscheidende Phänomene sind. Demselben Problem begegnen wir auch im Englischen und Kroatischen, so gibt es die im Englischen synonym benutzten Begriffe learning disability, learning difficulty, learning disorder, learning problems, poor learners und im Kroatischen die Begriffe teškoće učenja, poteškoće učenja, poremećaj učenja u. Ä. Dieses Durcheinander scheint zwar harmlos, doch macht es große Unterschiede im diagnostischen Verfahren. Auch das "Übersetzen“ von Diagnosen könnte wegen dieser Begriffsunterschiede zu Missverständnissen und im schlimmsten Falle zu Fehlbehandlungen führen. Um dies zu vermeiden und um die Differenzierung dieser Begriffe $\mathrm{zu}$ vereinfachen, werden immer wieder neue Definitionen und Abgrenzungen vorgeschlagen. Im Rahmen dieser Arbeit werden die Definitionsvorschläge von Gold (2011) verwendet, der meint, der Begriff Lernschwierigkeiten dient als Oberbegriff für alle Störungen und Ursachen, die das Lernen beeinträchtigen. An sich ist eine Lernschwierigkeit keine zu diagnostizierende Störung, Krankheit oder Behinderung, sondern die Folge bzw. Auswirkung. So gehören zu Lernschwierigkeiten z. B. die Dyslexie, die ADHS, aber auch eine leichte Intelligenzminderung, Seh- und Hörstörungen, fehlende Motivation, unangemessene Lernmöglichkeiten und alles, was sich negativ auf das Lernen auswirken könnte. Da die Lernschwierigkeit, wie schon gesagt, an sich keine Störung, sondern eine Folge ist, kann sie nicht diagnostiziert werden, sie wird festgestellt bzw. manifestiert. Bei Lernschwierigkeiten kann

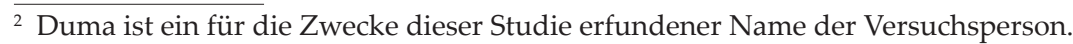


es sich beispielsweise um langsame und schwache Lerner handeln, aber auch um jene, die ihre Lernpotentiale nicht ausnutzen. Für Lernschwierigkeiten schwacher Lerner wird auch der Begriff Lernschwäche benutzt. Der einzige auffallende Unterschied zwischen Lernstörung und Lernschwäche ist das Diskrepanzkriterium ${ }^{3}$ - bei Lernschwächen ein Einfaches, bei Lernstörungen ein Doppeltes. Eine Lernschwäche liegt dann vor, wenn die Leistungen eines Schülers im Vergleich zu seinen Schulkameraden negativ auffällig sind. Für eine Diagnose der Lernstörungen bzw. der beschriebenen Entwicklungsstörungen schulischer Fertigkeiten ist wichtig, dass Ergebnisse trotz allgemeiner Intelligenz niedriger sind als jene der Schulkameraden. Sind beide Diskrepanzkriterien erfüllt, können folgende Lernstörungen diagnostiziert werden (s. International Statistical Classification of Diseases and Related Health Problems - ICD-104): Lesestörung und Lese-/ Rechtschreibstörung (F81.0), Isolierte Rechtschreibstörung (F81.1), Isolierte Rechenstörung (F81.2) und Kombinierte Störung schulischer Fertigkeiten (F81.3). Bei Lernbehinderung ${ }^{5}$ gibt es auch eine Intelligenzminderung, was die Lernbehinderung zu einer Teilmenge der Lernschwierigkeit (Gold, 2011: 11) macht.

\subsection{ADHS}

Obwohl es sich um eine der meist auftretenden „Störungen“ bei Kindern und Jugendlichenhandelt,meinendochviele,ADHSbzw.dieAufmerksamkeitsdefizitHyperaktivitätsstörung sei eine pharmaindustrieerzeugte Geldeinnahme oder wiederum das Ergebnis misslungener Erziehung. Daraus entwickeln sichfolgende Schwierigkeiten - in den letzten Jahren änderte sich nicht nur mehrmals die Bezeichnung für solch eine Störung, es gibt auch Unterschiede in der Begrifflichkeit bei verschiedenen Diagnosesystemen, bei denen auch immer wieder neue Symptome aufgenommen wurden, die vorherige und künftige Diagnosen beeinträchtigen. „Die Aufmerksamkeitsdefizit-/ Hyperaktivitätsstörung (ADHS) ist eine Bezeichnung für einen medizinischen Zustand, der eine gemischte Gruppe von störenden Verhaltensmustern beschreibt" (Holowenko, 1999: 19). Schon im Jahre 1890 wurden Symptome für solch einen Zustand von dem amerikanischen Philosophen und Psychologen William James, welcher oft als „Vater der modernen Psychologie“ (Holowenko,

\footnotetext{
${ }^{3}$ Die Diskrepanz ist eine Abweichung oder Differenz, die für eine Diagnoseerstellung erfüllt sein muss. Für die Lernschwäche genügt ein einfaches Diskrepanzkriterium bzw. das Bezugsgruppendiskrepanzkriterium: Die Leistungen werden in Bezug auf eine Altersgruppe gestellt, wobei sie deutlich schlechter sind als die der Bezugsgruppe (vgl. Tischler, 2017).

${ }^{4}$ https://icd.who.int/browse10/2016/en\#/F80 (11. 2. 2020)

5 „Eine Lernbehinderung [...] liegt vor, wenn bei schulpflichtigen Kindern lang andauernde, schwerwiegende und umfängliche Leistungsausfälle zu beobachten sind" (Walter und Wember, 2007: 3) und wenn ,[...] die allgemeine Schulleistungsschwäche zugleich mit einer Intelligenzminderung einhergeht (IQ < 85)“ (Gold, 2011: 11).
} 
1999: 17) bezeichnet wird, in seinen Principles of Psychology (Vol I and II) beschrieben. Heute ist es eine der meistvertretenen (Verhaltens-) Störungen bei Kindern. Laut Holowenko (1999) wird ihr Auftreten auf drei bis sechs Prozent aller Kinder in verschiedenen Kulturen und geographischen Regionen geschätzt, dabei sind Jungen in einem Verhältnis von ungefähr 3:1 überdurchschnittlich häufig vertreten. Die Störung umfasst auch die gesamte Lebensspanne und äußert sich je nach Alter und Geschlecht verschieden. So sind Jungen meist hyperaktive „Zappelmänner", Mädchen öfters zurückgezogene „Träumer".

Über die Ursachen einer ADHS wird derzeit noch immer diskutiert, dennoch ist es eindeutig, dass es sich selten um eine einzige handelt. Für die Entstehung einer ADHS sind meist mehrere zusammenwirkende Faktoren verantwortlich. $\mathrm{Zu}$ den meistbeschriebenen Ursachen zählen Genetik, neurologische und/oder biochemische Prozesse sowie Ursachen aus der Umwelt, wie z. B. ein dysfunktionales soziales Umfeld in Familie und/oder Schule (Holowenko, 1999: 21-29).

Die ärztliche Diagnose für ADHS versteht, dass die Verhaltensmuster Schwierigkeiten für das Kind in den Bereichen Entwicklung, Verhalten, Leistung, Familienbeziehungen und soziale Interaktion bedeuten. Dabei sind vor allem die drei Symptome Unaufmerksamkeit, Impulsivität und (jedoch nicht in allen Fällen) Hyperaktivität typisch (Holowenko, 1999: 19). Die Unaufmerksamkeit sei aber von Hyperaktivität-Impulsivität abzugrenzen: Unaufmerksamkeit kann als singuläres Symptom vorkommen, während Hyperaktivität und Impulsivität fast immer gemeinsam auftreten (Gawrilow, 2016: 21). Um jedoch Fehldiagnosen zu vermeiden, ist es wichtig, nicht nur die erwähnten Symptome in Betracht zu ziehen. Jedes Kind wird gelegentlich einige dieser Symptome aufzeigen - es wird manchmal unaufmerksam sein, impulsiv handeln, oder nicht still sitzen können, „aber ein gehäuftes Auftreten all dieser Probleme zusammen, die Intensität ihres Auftretens und wie sie während der Entwicklung eines Kindes existent bleiben, entscheidet wesentlich darüber, ob ein Kindheitssyndrom als ADHS identifiziert wird“ (Holowenko, 1999: 12).

Eine genaue Beschreibung der Kernsymptome ist in der Tabelle von Döpfner, Fröhlich und Lehmkuhl (2013) aufzufinden: 
Tabelle 1: Symptom-Kriterien der hyperkinetischen Störung nach ICD-10 (Forschungskriterien) und der Aufmerksamkeitsdefizit-/Hyperaktivitätsstörung nach DSM-IV (Döpfner, Fröhlich, Lehmkuhl, 2013: 1-2)

\begin{tabular}{|c|c|}
\hline A) Unaufmerksamkeit & $\begin{array}{l}\text { 1. Beachtet häufig Einzelheiten nicht oder macht Flüchtigkeitsfehler bei } \\
\text { den Schularbeiten, bei der Arbeit oder bei anderen Tätigkeiten. } \\
\text { 2. Hat oft Schwierigkeiten, längere Zeit die Aufmerksamkeit bei } \\
\text { Aufgaben oder Spielen aufrechtzuerhalten. } \\
\text { 3. Scheint häufig nicht zuzuhören, wenn andere ihn ansprechen. } \\
\text { 4. Führt häufig Anweisungen anderer nicht vollständig durch und kann } \\
\text { Schularbeiten, andere Arbeiten oder Pflichten am Arbeitsplatz nicht } \\
\text { zu Ende bringen (nicht aufgrund von oppositionellem Verhalten oder } \\
\text { Verständnisschwierigkeiten). } \\
\text { 5. Hat häufig Schwierigkeiten, Aufgaben und Aktivitäten zu } \\
\text { organisieren. } \\
\text { 6. Vermeidet häufig, hat eine Abneigung gegen oder beschäftigt } \\
\text { sich häufig nur widerwillig mit Aufgaben, die länger andauernde } \\
\text { geistige Anstrengungen erfordern (wie Mitarbeit im Unterricht oder } \\
\text { Hausaufgaben). } \\
\text { 7. Verliert häufig Gegenstände, die er/sie für Aufgaben oder Aktivitäten } \\
\text { benötigt (z. B. Spielsachen, Hausaufgabenhefte, Stifte, Bücher oder } \\
\text { Werkzeug). } \\
\text { 8. Lässt sich oft durch äußere Reize leicht ablenken. } \\
\text { 9. Ist bei Alltagstätigkeiten häufig vergesslich. }\end{array}$ \\
\hline B) Hyperaktivität & $\begin{array}{l}\text { 1. Zappelt häufig mit Händen oder Füßen oder rutscht auf dem Stuhl } \\
\text { herum. } \\
\text { 2. Steht \{häufig\} in der Klasse oder in anderen Situationen auf, in denen } \\
\text { Sitzenbleiben erwartet wird. } \\
\text { 3. Läuft häufig herum oder klettert exzessiv in Situationen, in denen dies } \\
\text { unpassend ist (bei Jugendlichen oder Erwachsenen kann dies auf ein } \\
\text { subjektives Unruhegefühl beschränkt bleiben). } \\
\text { 4. Hat häufig Schwierigkeiten, ruhig zu spielen oder sich mit } \\
\text { Freizeitaktivitäten ruhig zu beschäftigen. } \\
\text { 5. \{Ist häufig ,auf Achse“ oder handelt oftmals, als wäre er "getrieben“.\} } \\
\text { 6. (Zeigt ein anhaltendes Muster exzessiver motorischer Aktivität, } \\
\text { das durch die soziale Umgebung oder durch Aufforderungen nicht } \\
\text { durchgreifend beeinflussbar ist.) }\end{array}$ \\
\hline C) Impulsivität & $\begin{array}{l}\text { 1. Platzt häufig mit der Antwort heraus, bevor die Frage zu Ende gestellt } \\
\text { ist. } \\
\text { 2. Kann häufig nur schwer warten, bis er/sie an der Reihe ist (bei Spielen } \\
\text { oder in Gruppensituationen). } \\
\text { 3. Unterbricht und stört andere häufig (platzt z. B. in Gespräche oder in } \\
\text { Spiele anderer hinein). } \\
\text { 4. Redet häufig übermäßig viel (ohne angemessen auf soziale } \\
\text { Beschränkungen zu reagieren). \{Im DSM-IV unter Hyperaktivität } \\
\text { subsumiert.\} }\end{array}$ \\
\hline
\end{tabular}

Anmerkung: \{\}$=$ nur DSM-IV; ( ) = nur ICD-10

Welche dieser Symptomkriterien auftreten und in welcher Anzahl sie unter Unaufmerksamkeit, Hyperaktivität und Impulsivität vorhanden sind, wird bestimmen, welcher ADHS-Subtyp diagnostiziert wird. So müssen z. B. mindestens sechs Kriterien unter Aufmerksamkeit, drei unter Hyperaktivität, und mindestens eines unter Impulsivität erfüllt sein, damit 
eine hyperkinetische Störung des Sozialverhaltens festgestellt werden kann, wobei auch die Kriterien für eine Störung des Sozialverhaltens erfüllt sein müssen (siehe auch Abbildung 1).

Es soll zudem erwähnt werden, dass ADHS im Rahmen zweier Klassifikationssysteme diagnostiziert wird - das sind das DSM (engl. Diagnostic and Statistical Manual of Mental Disorders, dt. Diagnostisches und statistisches Handbuch psychischer Störungen) und die schon angeführte ICD10 (engl. International Statistical Classification of Diseases and Related Health Problems, dt. Internationale statistische Klassifikation für Krankheiten und verwandte Gesundheitsprobleme).

Dass die ADHS oft als eine "Modediagnose“ beschrieben wird, ist Beweis dafür, wie häufig sie diagnostiziert wird. Für die Validität dieser Störung spricht dennoch die Tatsache, dass sie weltweit in unterschiedlichen Kulturen erscheint. In Deutschland haben laut einer KiGGS-Studie ${ }^{6}$, über die Gawrilow (2016: 47) berichtet, 4,8 \% der Kinder und Jugendlichen eine von einem Psychologen diagnostizierte ADHS. Dabei beträgt die Prävalenz 1,5 \% im Vorschulalter, 5,3 \% im Grundschulalter, 7,1 \% bei 11-13-Jähigen und 5,6 \% bei 14-17-Jährigen. „ADHS wird weiterhin laut der KiGGS-Studie häufiger bei Kindern aus Familien mit niedrigem sozioökonomischen Status im Vergleich zu Familien mit einem mittleren oder hohen sozioökonomischen Status diagnostiziert" (Gawrilow, 2016: 47-48). Auch zeigt die Studie, „dass Familien mit Migrationshintergrund seltener über eine ADHS-Diagnose als Familien ohne Migrationshintergrund berichten. Jedoch gibt es mehr ADH-Verdachtsfälle bei Familien mit als ohne Migrationshintergrund" (Gawrilow, 2016: 48). Daraus ergibt sich, dass sich Kinder aus Familien mit Migrationshintergrund durch die ADHS-Symptome öfter auffällig machen, doch nehmen ihre Familien seltener Diagnose, Beratung und Intervention in Anspruch.

\subsection{Dyslexie}

Auch bei der Dyslexie gibt es Unterschiede und Schwierigkeiten in der Begrifflichkeit, denn es können allerlei Subtypen von Dyslexie je nach Ursache, Symptomatik und sogar abhängig davon, welchen Sprachbereich sie betrifft, festgestellt werden. Auch unterscheiden sich die Begriffe je nach Sprachraum - im deutschsprachigen Raum wird die Dyslexie oft Legasthenie oder Lese-Rechtschreibschwäche (LRS) genannt. Diese Begriffe scheinen jedoch nicht sehr praktisch - dass es sich um eine "Schwäche" handelt, signalisiert sofort, dass bei einer Lese-Rechtschreibschwäche ein einfaches Diskrepanzkriterium ausreicht und dass solch eine „Schwäche“ in der ICD-

\footnotetext{
${ }^{6}$ Die KiGGS-Studien sind große, umfassende Studien des Robert-Koch-Instituts, die Fragen zur Gesundheit von Kindern und Jugendlichen in Deutschland erforschen.
} 
10 nicht aufgefunden wird. Des Weiteren reden Miles und Miles im Buch Dislexia - A Hundert Years on (1999) von zwei Subtypen von Dyslexie - dem auditiven und dem visuellen Subtyp. Der Unterschied wird folgendermaßen beschrieben:

Das Kind lernt das gesprochene Wort und wie die Buchstaben aussehen, es kann aber diese Bilder nicht mit der Art und Weise, wie sie klingen, verbinden (auditiver Typ der Dyslexie). Es mag auch das Gegenteil erscheinen. Es lernt, wie die Buchstaben klingen, kann aber keine normale Verbindung zwischen diesen akustischen Bildern und ihrem Aussehen herstellen. (Miles und Miles, 1999: 95)

Der auditive und der visuelle Typ erscheinen also in Bezug auf das Problemfeld. Nehmen wir die Ursache als Kriterium, so sprechen wir von der Erworbenen Dyslexie (engl. aquired dyslexia), erworben durch Hirnschädigungen wiez. B. einenSchlaganfall, oderder Entwicklungsdyslexie (engl. developmental dyslexia), die genetisch veranlagt ist. Auch in der ICD-10 können zwei Typen von Dyslexie vorgefunden werden - unter dem Code R48.0 gehört sie zur Übergruppe „Dyslexieund sonstige Werkzeugstörungen, anderenorts nicht klassifiziert" (R48.-). Als Entwicklungsdyslexie wird sie in der ICD-10 unter dem Code F81.0 vermerkt. Fortan wird von der Entwicklungsdyslexie gesprochen.

Die Ursache der Dyslexie ist das Defizit phonologischer Komponenten der Sprache;sie ist also neurologischen Ursprungs und zeichnet sich durch Schwierigkeiten mit genauer und/oder fließender Worterkennung und durch schlechte Schreib- und Decodierfähigkeit aus (International Dyslexia Association, 20177). Ein Kind mit Dyslexie wird wahrscheinlich Schwierigkeiten in folgenden Bereichen aufzeigen: beim Erlernen von Buchstaben und ihrer Laute, bei der Organisation von geschriebener und gesprochener Sprache, beim Merken von Zahlenfakten, beim Mithalten und Verstehen von längeren Leseaufträgen, bei der Rechtschreibung, bei der korrekten Ausführung mathematischer Operationen und beim Erlernen einer Fremdsprache (International Dyslexia Association, 2017: 4)

\subsection{Leichte Intelligenzminderung}

Die leichte Intelligenzminderung (engl. mild mental retardation) gehört zu den Intelligenzstörungen (engl. mental retardation). Dies sind Zustände verzögerter oder unvollständiger Entwicklung geistiger Fertigkeiten wie Kognition, Sprache, motorische und soziale Fähigkeiten. Der Schweregrad einer Intelligenzstörung wird anhand standardisierter Intelligenztests von einem Diagnostiker festgestellt. Intellektuelle Fähigkeiten und soziale

\footnotetext{
$\overline{7}$ https://dyslexiaida.org/wp-content/uploads/2015/01/DITC-Handbook.pdf (17. 8. 2018)
} 
Anpassung können sich, wenn auch nur in geringem Maße, durch Übung und Rehabilitation verbessern.

Eine leichte Intelligenzminderung (F70) ${ }^{8}$ liegt vor, wenn der IQ-Bereich einer Person zwischen 50-69 liegt. Dies ist bei Erwachsenen mit einem Intelligenzalter von 9 bis 12 Jahren gleichzustellen. Viele Erwachsene können zwar arbeiten und ihren Beitrag zur Gesellschaft leisten, sie wirkt sich bei Kindern aber in Lernschwierigkeiten in der Schule aus. Sollte neben der Intelligenzminderung Komorbidität eines anderen Zustandsbildes, wie andere Entwicklungsstörungen, Störungen des Sozialverhaltens usw. vorliegen, müssen zusätzliche Schlüsselnummern (F70.0 - F70.9) benutzt werden, die zusätzliche Beobachtung oder Behandlung erfordern.

\subsection{Sprachentwicklungsstörung}

Auch bei der Sprachentwicklungsstörung finden wir unterschiedliche Begriffe - Sprachentwicklungsstörung, -behinderung oder im Englischen specific language impairment. In diesem Beitrag ist die Rede von der Sprachentwicklungsstörung welche in der ICD-10 unter dem Code F80.- und Codenamen „Umschriebene Entwicklungsstörung des Sprechens unter der Sprache" aufgefunden wird. Solche Entwicklungsstörungen werden gemäß der ICD-10 folgendermaßen definiert:

Es handelt sich um Störungen, bei denen die normalen Muster des Spracherwerbs von frühen Entwicklungsstadien an beeinträchtigt sind. Die Störungen können nicht direkt neurologischen Störungen oder Veränderungen des Sprachablaufs, sensorischen Beeinträchtigungen, Intelligenzminderung oder Umweltfaktoren zugeordnet werden. Umschriebene Entwicklungsstörungen des Sprechens und der Sprache ziehen oft sekundäre Folgen nach sich, wie Schwierigkeiten beim Lesen und Rechtschreiben, Störungen im Bereich der zwischenmenschlichen Beziehungen, im emotionalen und Verhaltensbereich. ${ }^{9}$

Da Sprachentwicklungsstörungen aufgrund sehr verschiedener Ursachen entstehen können, unterscheidet man meist zwischen zwei Subtypen den spezifischen Sprachentwicklungsstörungen und den allgemeinen Sprachentwicklungsstörungen. Eine spezifische Sprachentwicklungsstörung liegt vor, wenn ein Kind erhebliche Schwierigkeiten in der Sprache hat und sprachauffällig ist, obwohl keine vorausgehenden organischen, mentalen oder emotionalen Schädigungen bestehen, was bedeutet, dass das Kind gut hören und sehen kann, sich geistig und körperlich normal entwickelt und ihm das Elternhaus optimale Entwicklungsbedingungen bietet (Wendlandt, 2006). Dazu gehören Auffälligkeiten und Schwierigkeiten mit Aussprache

\footnotetext{
8 s. ICD-10 https://www.icd-code.de/suche/icd/code/F70.-.html?sp=SF70 (10. 9. 2018)

9 http://www.icd-code.de/suche/icd/code/F80.-.html?sp=Ssprachst\%F6rung (10. 9. 2018)
} 
(Dyslalie), Sprachverständnis, Grammatik (Dysgrammatismus; Satzbau und Wortendung), wie auch ein eingeschränkter Wortschatz und eingeschränkte kommunikative und dialogische Fähigkeiten. Eine allgemeine Sprachentwicklungsstörung hingegen liegt vor, wenn organische, mentale oder emotionale Schädigungen, wie z. B. Hör- und Sehbeeinträchtigungen, Intelligenzminderung oder emotionale Belastung bestehen und die Sprachentwicklung stören, wobei sich die Symptome nicht weiter von den spezifischen Sprachentwicklungsstörungen unterscheiden (ebd.).

In Tabelle 2 wird die Auswirkung der spezifischen Sprachstörung auf Sprachfähigkeit mit denen der Dyslexie, der ADHS und der leichten Intelligenzminderung verglichen.

Tabelle 2: Auswirkung der spezifischen Sprachstörung auf Sprachfähigkeit (übernommen und übersetzt aus Galić-Jurušić, 2004)

\begin{tabular}{|c|c|c|c|c|}
\hline $\begin{array}{l}\text { Störung/ } \\
\text { Typ der Schwä- } \\
\text { che }\end{array}$ & $\begin{array}{l}\text { Kinder mit Dys- } \\
\text { lexie }\end{array}$ & $\begin{array}{l}\text { Kinder mit spezi- } \\
\text { fischen Sprach- } \\
\text { störungen }\end{array}$ & $\begin{array}{l}\text { Kinder mit Auf- } \\
\text { merksamkeitsde- } \\
\text { fizit/ ADHS }\end{array}$ & $\begin{array}{l}\text { Kinder mit leich- } \\
\text { ter Intelligenz- } \\
\text { minderung }\end{array}$ \\
\hline $\begin{array}{l}\text { Sprachschwieri- } \\
\text { gkeiten }\end{array}$ & $\begin{array}{l}\text { Schwierigkeiten } \\
\text { mit dem Laute- } \\
\text { system, erschwer- } \\
\text { te Unterschei- } \\
\text { dung ähnlich } \\
\text { klingender Laute, } \\
\text { andere Sprach- } \\
\text { komponenten } \\
\text { altersentsprech- } \\
\text { end entwickelt, } \\
\text { fortgeschrittene } \\
\text { konzeptionelle } \\
\text { Entwicklung, } \\
\text { finden Wege ihre } \\
\text { Gedanken } \\
\text { sprachlich } \\
\text { auszudrücken }\end{array}$ & $\begin{array}{l}\text { Schwierigkeiten } \\
\text { in allen Sprach- } \\
\text { komponenten, } \\
\text { dysgramma- } \\
\text { tisches Sprach- } \\
\text { system, } \\
\text { konzeptionelle } \\
\text { Entwicklung } \\
\text { etwas langsa- } \\
\text { mer, finden nur } \\
\text { schwer Wege ihre } \\
\text { Gedanken } \\
\text { sprachlich } \\
\text { auszudrücken }\end{array}$ & $\begin{array}{l}\text { Mögliche Schwie- } \\
\text { rigkeiten in allen } \\
\text { Sprachkompo- } \\
\text { nenten; Schwie- } \\
\text { rigkeiten mit } \\
\text { phonologischen } \\
\text { Komponenten } \\
\text { betont, wenn die } \\
\text { Aufmerksam- } \\
\text { keitsstö-rung } \\
\text { aufgrund } \\
\text { Dyslexieentwick- } \\
\text { lung abläuft; } \\
\text { Agrammatismus } \\
\text { oder langsamere } \\
\text { Sprachentwick- } \\
\text { lung, wenn das } \\
\text { Aufmerksam- } \\
\text { keitsdefizit an } \\
\text { Sprachschwie- } \\
\text { rigkeiten oder } \\
\text { verminderten } \\
\text { kognitiven Fähig- } \\
\text { keiten grenzt }\end{array}$ & $\begin{array}{l}\text { Mögliche } \\
\text { Schwierigkeiten } \\
\text { in allen Sprach- } \\
\text { komponenten } \\
\text { mit ernsthaften } \\
\text { Schwierigkeiten } \\
\text { bei Konzepter- } \\
\text { stellung, der } \\
\text { Entwicklung von } \\
\text { Sätzen, chaotische } \\
\text { oder fehlende } \\
\text { Ideen erlauben } \\
\text { keine einfache } \\
\text { Ausdrucksweise }\end{array}$ \\
\hline
\end{tabular}




\begin{tabular}{|c|c|c|c|c|}
\hline $\begin{array}{l}\text { Störung/ } \\
\text { Typ der Schwä- } \\
\text { che }\end{array}$ & $\begin{array}{l}\text { Kinder mit Dys- } \\
\text { lexie }\end{array}$ & $\begin{array}{l}\text { Kinder mit spezi- } \\
\text { fischen Sprach- } \\
\text { störungen }\end{array}$ & $\begin{array}{l}\text { Kinder mit Auf- } \\
\text { merksamkeitsde- } \\
\text { fizit/ ADHS }\end{array}$ & $\begin{array}{l}\text { Kinder mit leich- } \\
\text { ter Intelligenz- } \\
\text { minderung }\end{array}$ \\
\hline $\begin{array}{l}\text { Kognitive } \\
\text { Schwierigkeiten }\end{array}$ & $\begin{array}{l}\text { entwickeln sich } \\
\text { kognitiv gut, ha- } \\
\text { ben Resonanzpo- } \\
\text { tential, betrachten } \\
\text { ein Problem gut } \\
\text { von unterschied- } \\
\text { lichen Seiten, } \\
\text { ändern leicht die } \\
\text { Sichtweise, haben } \\
\text { keine Probleme } \\
\text { mit Konzentra- } \\
\text { tionstiefe und } \\
\text { leicht entnom- } \\
\text { mener Aufmerk- } \\
\text { samkeit, denken } \\
\text { logisch nach, } \\
\text { gutes Verbinden } \\
\text { von konzeptio- } \\
\text { nellen Sets }\end{array}$ & \begin{tabular}{|l|} 
haben ein gutes \\
kognitives Po- \\
tential, es fehlen \\
ihnen manchmal \\
die Worte für \\
kognitive Kate- \\
gorien, welche sie \\
verstehen (Zeit, \\
Raum), durch \\
richtige Sprach- \\
auswahl gesteuert \\
räsonieren sie gut \\
und verbinden \\
gut konzeptionel- \\
le Sets
\end{tabular} & \begin{tabular}{|l|} 
mögliche \\
Schwierigkeiten \\
in Kognition \\
aufgrund der \\
fehlenden Selek- \\
tivität und des \\
beschleunigten \\
oder verlangsam- \\
ten Bewusstseins- \\
flusses, chaotisch \\
und unlogisch \\
aufgrund hoher \\
Geschwindigkeit, \\
Ungenaue Wahr- \\
nehmung kann \\
den falschen \\
Eindruck eines \\
geringeren kog- \\
nitiven Potenzials \\
vermitteln, akzen- \\
tuierte Inkonsis- \\
tenz in kognitiven \\
Bereichen
\end{tabular} & $\begin{array}{l}\text { Schwierigkeiten } \\
\text { in der kognitiven } \\
\text { Entwicklung } \\
\text { fast die Regel, } \\
\text { unlogisches } \\
\text { Denken, leicht } \\
\text { abzulenkende } \\
\text { Aufmerksam- } \\
\text { keit ohne einen } \\
\text { tieferen Fokus, } \\
\text { Schwierigkeiten } \\
\text { beim Merken } \\
\text { und Verbinden, } \\
\text { eine ausgeprägte } \\
\text { Verlangsamung } \\
\text { der konzeptionel- } \\
\text { len Entwicklung, } \\
\text { unzureichende } \\
\text { Geschwindigkeit } \\
\text { und Präzision der } \\
\text { Wahrnehmung }\end{array}$ \\
\hline $\begin{array}{l}\text { Schwierigkeiten } \\
\text { im Lesen und } \\
\text { Schreiben }\end{array}$ & $\begin{array}{l}\text { Schwierigkeiten } \\
\text { bei der Deko- } \\
\text { dierung, Ver- } \\
\text { wechslung von } \\
\text { Buchstaben durch } \\
\text { visuelle und } \\
\text { akustische Ähn- } \\
\text { lichkeit, das Po- } \\
\text { tential Kompen- } \\
\text { sationsstrate-gien } \\
\text { zu schaffen } \\
\text { und dann gutes } \\
\text { Verständnis, } \\
\text { eine ungeformte } \\
\text { Handschrift, der } \\
\text { schriftliche Aus- } \\
\text { druck entwickelt } \\
\text { sich stark durch } \\
\text { das Erlernen der } \\
\text { Schriftsprache }\end{array}$ & $\begin{array}{l}\text { kleinere Schwie- } \\
\text { rigkeiten bei der } \\
\text { Dekodierung } \\
\text { als Kinder mit } \\
\text { Dyslexie, } \\
\text { Verständnis- } \\
\text { schwierigkeiten } \\
\text { sind etwas aus- } \\
\text { geprägter, beim } \\
\text { Schreiben können } \\
\text { sie eine präzise, } \\
\text { schön geformte } \\
\text { Handschrift ent- } \\
\text { wickeln, haben } \\
\text { Schwierigkeiten } \\
\text { die Gedanken } \\
\text { schriftlich in } \\
\text { einem Aufsatz } \\
\text { auszudrücken, } \\
\text { können aber } \\
\text { Fortschritte im } \\
\text { Lernen, vor allem } \\
\text { in der Schriftspra- } \\
\text { che, machen }\end{array}$ & $\begin{array}{l}\text { mildere Art von } \\
\text { Dekodierungs- } \\
\text { schwie-rigkeiten, } \\
\text { Verständnis- } \\
\text { schwierigkei- } \\
\text { ten aufgrund } \\
\text { hereinplatzen- } \\
\text { der Gedanken, } \\
\text { welche nicht mit } \\
\text { dem Text zu tun } \\
\text { haben, Schwie- } \\
\text { rigkeiten mit } \\
\text { dem Fokussieren, } \\
\text { haben eine große } \\
\text { Abneigung, mit } \\
\text { dem Lesen anzu- } \\
\text { fangen, können } \\
\text { die Lesetechnik } \\
\text { gut entwickeln, } \\
\text { Schrift unge- } \\
\text { formt, das Schrei- } \\
\text { ben chaotisch }\end{array}$ & $\begin{array}{l}\text { sie beherrschen } \\
\text { allmählich die } \\
\text { Lesetechnik, kön- } \\
\text { nen langsam mit } \\
\text { relativ wenigen } \\
\text { Fehlern lesen, } \\
\text { das Verständnis } \\
\text { ist erschwert, } \\
\text { das Schreiben ist } \\
\text { inhaltlich knapp, } \\
\text { die Handschrift } \\
\text { ist lange Zeit } \\
\text { bemerkbar unent- } \\
\text { wickelt }\end{array}$ \\
\hline
\end{tabular}




\begin{tabular}{|c|c|c|c|c|}
\hline $\begin{array}{l}\text { Störung/ } \\
\text { Typ der Schwä- } \\
\text { che }\end{array}$ & $\begin{array}{l}\text { Kinder mit Dys- } \\
\text { lexie }\end{array}$ & $\begin{array}{l}\text { Kinder mit spezi- } \\
\text { fischen Sprach- } \\
\text { störungen }\end{array}$ & $\begin{array}{l}\text { Kinder mit Auf- } \\
\text { merksamkeitsde- } \\
\text { fizit/ ADHS }\end{array}$ & $\begin{array}{l}\text { Kinder mit leich- } \\
\text { ter Intelligenz- } \\
\text { minderung }\end{array}$ \\
\hline $\begin{array}{l}\text { Fortschritte in } \\
\text { Lernfähigkeiten }\end{array}$ & $\begin{array}{l}\text { Kompensations- } \\
\text { strategien des } \\
\text { Lernens akzen- } \\
\text { tuiert, durch } \\
\text { Unterrichtung } \\
\text { können sie Stoff } \\
\text { gut organisieren, } \\
\text { neigen zu krea- } \\
\text { tiven visuellen } \\
\text { Lösungen, in } \\
\text { Mathe werden } \\
\text { Verfahrensdetails } \\
\text { ausgelassen, das } \\
\text { Merken von Halb- } \\
\text { resultaten, sehen } \\
\text { das Ganze gut ein } \\
\text { und haben Ideen } \\
\text { für das Lösen } \\
\text { von Aufgaben, } \\
\text { verstehen genaue } \\
\text { Konzepte gut, } \\
\text { merken sich } \\
\text { schwer Namen, } \\
\text { ersetzen dies } \\
\text { durch Mnemo- } \\
\text { techniken, besser } \\
\text { in mündlichen } \\
\text { Antworten }\end{array}$ & $\begin{array}{l}\text { zeigen Gelerntes } \\
\text { schwer, } \\
\text { geben einen } \\
\text { schlechteren Ein- } \\
\text { druck von ihrem } \\
\text { Wissen, finden } \\
\text { kaum Worte und } \\
\text { Konstrukte, die } \\
\text { ein wahres Bild } \\
\text { ihres Wissens } \\
\text { wiedergeben } \\
\text { können, gleicher- } \\
\text { maßen schwierig } \\
\text { Wissen sowohl } \\
\text { in mündlicher als } \\
\text { auch in schrift- } \\
\text { licher Form zu } \\
\text { zeigen, Schwie- } \\
\text { rigkeiten beim } \\
\text { Verständnis der } \\
\text { mathematischen } \\
\text { Sprache, gut in } \\
\text { mathematischen } \\
\text { Verfahren, kön- } \\
\text { nen die Fähigkei- } \\
\text { ten des unabhän- } \\
\text { gigen Lernens } \\
\text { erreichen }\end{array}$ & $\begin{array}{l}\text { Ungewissheit in } \\
\text { Fortschritten aller } \\
\text { Lernfähigkeiten, } \\
\text { alle möglichen } \\
\text { Resultate ab- } \\
\text { hängig von der } \\
\text { Möglichkeit der } \\
\text { Kompensations- } \\
\text { fähigkeit, die } \\
\text { Schwierigkeiten } \\
\text { in der Präzision } \\
\text { der Perzeption, } \\
\text { der Ausdauer } \\
\text { und der Tiefe der } \\
\text { Aufmerksamkeit } \\
\text { zu überwinden, } \\
\text { Schwierigkeiten } \\
\text { mit Mathema- } \\
\text { tik, können die } \\
\text { Fähigkeiten des } \\
\text { unabhängigen } \\
\text { Lernens nur } \\
\text { schwer entwi- } \\
\text { ckeln }\end{array}$ & $\begin{array}{l}\text { abhängig von } \\
\text { umfangreicher } \\
\text { Hilfe, Schwierig- } \\
\text { keiten, die Idee } \\
\text { der Zahl zu meis- } \\
\text { tern, desorientiert } \\
\text { in der Abfolge } \\
\text { mathemati- } \\
\text { scher Verfahren } \\
\text { beim Rechnen. } \\
\text { Schwierigkeiten } \\
\text { beim Meistern } \\
\text { von komplexeren } \\
\text { Konzepten, Ge- } \\
\text { dächtnisschwie- } \\
\text { rigkeiten machen } \\
\text { es unmöglich, } \\
\text { Fakten zu ver- } \\
\text { binden und eine } \\
\text { feste Grundlage } \\
\text { für die Kennt- } \\
\text { nis der meisten } \\
\text { Lehrinhalte zu } \\
\text { schaffen. }\end{array}$ \\
\hline $\begin{array}{l}\text { Optimaler Schul- } \\
\text { ansatz }\end{array}$ & $\begin{array}{l}\text { Individualisierter } \\
\text { Ansatz zum regu- } \\
\text { lären Unterrichts- } \\
\text { programm }\end{array}$ & $\begin{array}{l}\text { Individualisierter } \\
\text { Ansatz zum regu- } \\
\text { lären Unterrichts- } \\
\text { programm }\end{array}$ & $\begin{array}{l}\text { Individualisier- } \\
\text { ter Ansatz zum } \\
\text { regulären Unter- } \\
\text { richtsprogramm } \\
\text { / Sonderpädago- } \\
\text { gische Förderung } \\
\text { im regulären } \\
\text { Unterricht }\end{array}$ & $\begin{array}{l}\text { Sonderpädagogi- } \\
\text { sche Förderung } \\
\text { im regulären } \\
\text { Unterricht / Aus- } \\
\text { bildung an einer } \\
\text { Sonderpädagogi- } \\
\text { schen Institution }\end{array}$ \\
\hline
\end{tabular}

\section{KINDER MIT MEHREREN LENRSCHWIERIGKEITEN - KOMORBIDITÄT}

Das Online Magazin für Naturwissenschaft und Technik „Spektrum“10 definiert Komorbidität als Anwesenheit von mehr als einer (psychischen) Störung in einer Person in einem bestimmten zeitlichen Rahmen. Das bedeutet, dass sich neben der Grunderkrankung bei Patienten weitere Erkrankungen melden. "Spektrum" erklärt weiter, dass in der klinisch-psychologischen Fachliteratur folgende Arten von Komorbidität unterschieden werden - die

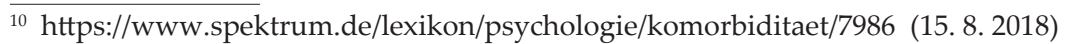


„,interne Komorbidität“ bzw. das gemeinsame Auftreten von Unterkategorien der gleichen Störungsgruppe und die „externe Komorbidität", das gemeinsame Auftreten von psychischen Störungen verschiedener Störungskategorien. So wäreein Beispiel für interne Komorbidität z. B., wennein Patient unter mehreren Untergruppen der Entwicklungsstörungen (s. ICD-10 die Codes F80-F89) leidet, z. B. unter der Umschriebenen Entwicklungsstörung des Sprechens und der Sprache (F80.-) und der Umschriebenen Entwicklungsstörung schulischer Fertigkeiten (F81.-). Bei einer externen Komorbidität handelt es sich also um Störungen unterschiedlicher Kategorien, so wäre ein Beispiel dafür ein Patient mit Schizophrenie (F20.-) und Vaskulärer Demenz (F01.-).

Komorbidität trifft nicht selten auf. Eine der möglichen Ursachen für Komorbidität ist z. B., so "Spektrum", eine gemeinsame Etymologie der Störungen. Dasselbe gilt auch für Komorbidität von Lernschwierigkeiten; so zeigen sich bei einem Diagnoseverfahren oft mehrere komorbide Lernstörungen und es wird nur schwer festgestellt, ob sie parallel entstanden sind oder ob die eine die Entstehung der anderen verursachte. Dies ist auch einer der Gründe, warum es oft zu Fehldiagnosen kommt bzw. warum es oft schwer ist, eine endgültige Diagnose aufzustellen. Die ADHS ist hier keine Ausnahme. So beschreibt Gawrilow (2016) in ihrem Buch, dass ADHS-Betroffene oft komorbide Störungen aufweisen - es handelt sich hier öfters um die Regel als die Ausnahme: „Komorbide Störungen sind bei der ADHS die Regel: Insgesamt weisen ca. 2/3 der Kinder mit ADHS neben den Kernsymptomen für die ADHS noch weitere Störungen auf. Sobald komorbide Störungen im Spiel sind, wird eine ADHS-Diagnose erheblich erschwert" (Gawrilow, 2016: 31-32). Zu den häufigsten komorbiden Störungen der ADHS gehören externalisierende Störungen, internalisierende Störungen, sowie Lern- und Leistungsstörungen. Kinder mit schulischen Minderleistungen, Lernschwächen und Lernstörungen zeigen größere Anfälligkeit für externalisierende und internalisierende Störungen und Problemverhalten, was auch zu erwarten ist. Muss sich ein Kind alltäglich mit Misserfolgen auseinandersetzen, ist zu erwarten, dass es mit der Zeit Situationen vermeiden will, aus welchen diese Misserfolge entspringen. Aus Misserfolgserfahrungen werden Misserfolgserwartungen, sprich Angst, und führen weiterhin zu Depression und Entmutigung. Für Kinder mit Lernschwierigkeiten ist es von höchster Wichtigkeit, dass sie eine positive Einstellung dem Lernen gegenüber behalten, viel Motivation und eine starke Unterstützung von Eltern und Lehrern bekommen.

\section{TESTMETHODEN UND VERFAHREN}

Jeder Lehrer hat die Möglichkeit, die Lernfähigkeiten und das Sprachniveau der Schüler mit Schwierigkeiten einigermaßen $\mathrm{zu}$ testen, um die Unterrichtsstunden besser zu gestalten oder zu personalisieren. Wenn nötig, 
kann man auch die ADHS Diagnose mittels einer Checkliste testen, um diese Lernschwierigkeit besser zu verstehen. Eine frei verwendbare Checkliste ist die nach Döpfner, Görtz-Dorten und Lehmkuhl (2008) (s. Kapitel 4.1.). Mithilfe des MoCA-Tests (s. Kapitel 4.2.) kann man auch kognitive Defizite feststellen. Das Sprachniveau ist durch Muster-Sprachniveautests, beispielsweise der international anerkannten, getesteten und validierten ECL (European Consortium for the Certificate of Attainment in Modern Languages)Tests $^{11}$, zu bestimmen. Zur Feststellung der Lernstile der Schüler kann man den Fragebogen von Education Planner (s. Kapitel 4.3.) einsetzen. Die genannten Testmethoden und Verfahren wurden auch in der Fallstudie benutzt, die in diesem Beitrag dargestellt wird (s. Kapitel 5.)

\subsection{ADHS Diagnose-Checkliste}

Die ADHS Diagnose-Checkliste ${ }^{12}$ nach Döpfner, Görtz-Dorten und Lehmkuhl (2008) beinhaltet klare Kriterien für die Subtypen der ADHS und kann von jedem Lehrer selbst durchgeführt werden (Abb. 1). Die Checkliste besteht aus jeweils drei Einheiten für die drei Kernsymptome Aufmerksamkeit, Überaktivität bzw. Hyperaktivität, und Impulsivität. Für das Kernsymptom Aufmerksamkeit werden neun Symptomkriterien beschrieben, für die Hyperaktivität fünf und für die Impulsivität vier.

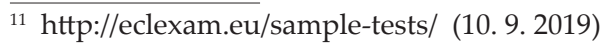

12 https://www.zentrales-adhs-netz.de/fileadmin/redakteure/zentrales_adhs-netz/f\%C3\%BCr_therapeuten/Diagnostik/Diagnostik_Ki_Ju/Diagnose_Checkliste_DCL_AD̄HS.pdf

(7. 10. 2018)
} 


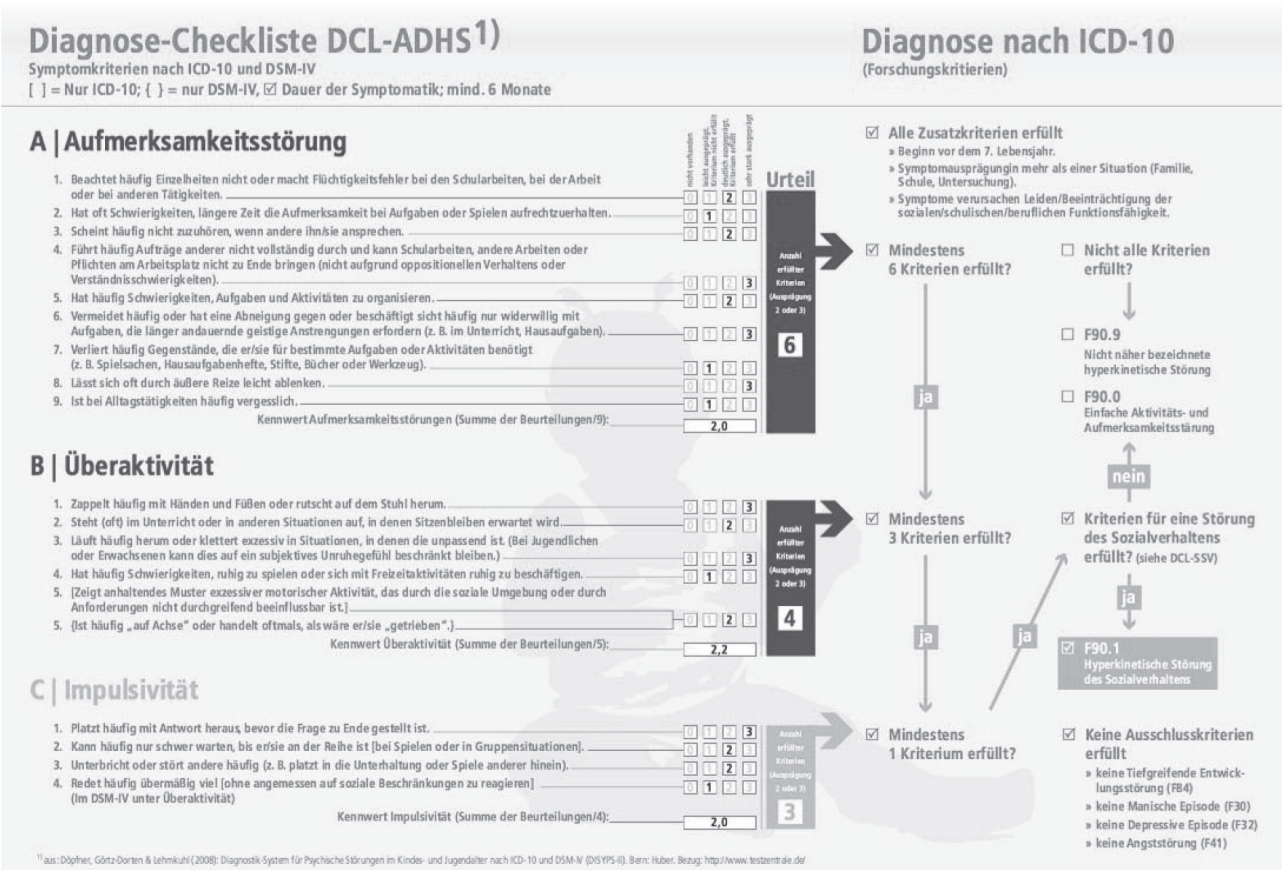

Abbildung 1: Diagnose-Checkliste DCL-ADHS nach Döpfner, Görtz-Dorten und Lehmkuhl (2008)

\subsection{MoCA-Test}

Der Montreal Cognitive Assessment ${ }^{13}$ (MoCA) Test ist ein Screening-Instrument für die leichte Intelligenzminderung und ist in mehreren Sprachen verfügbar. Er berücksichtigt unterschiedliche kognitive Bereiche: Aufmerksamkeit und Konzentration, Exekutivfunktionen, Gedächtnis, Sprache, visuokonstruktive Fähigkeiten, konzeptuelles Denken, Rechnen und Orientierung ${ }^{14}$. Die Durchführungszeit beträgt ungefähr 10 Minuten. Hier eine verkleinerte Abbildung:

${ }^{13}$ https://www.mocatest.org (7. 9. 2018)

14 https://www.memoryclinic.ch/de/main-navigation/neuropsychologen/weitere-testverfahren/ (10. 9. 2018) 


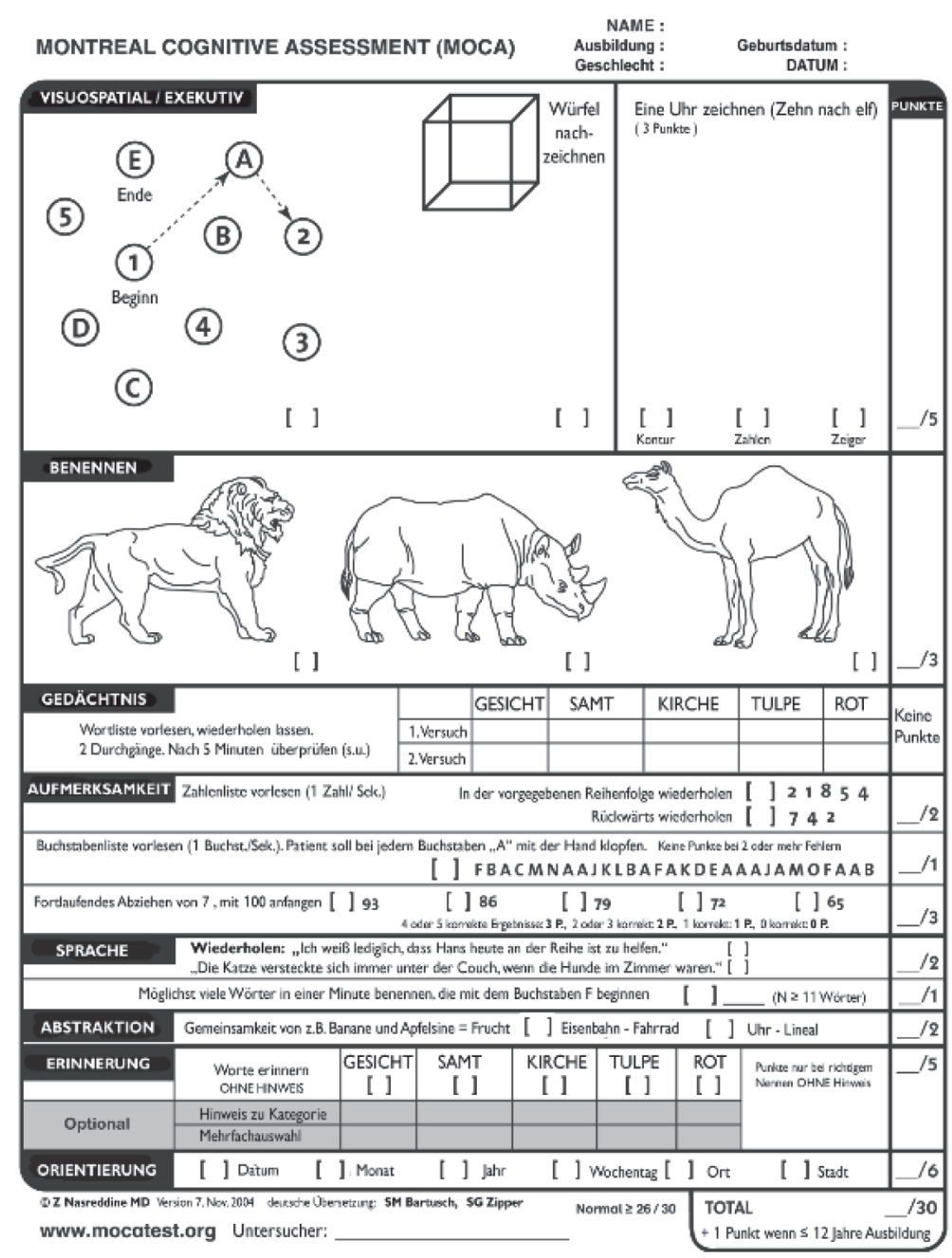

Abbildung 2: MoCA-Test für Intelligenzminderung

Die gesamte Punktezahl, die im MoCA-Test erzielt werden kann, beträgt 30 Punkte, wobei ein Punkt zugerechnet wird, wenn die getestete Person weniger als 12 Jahre Ausbildung hatte. Bei einer erzielten Punktezahl von 26 oder mehr Punkten liegt keine Intelligenzminderung vor. Bei 25 Punkten oder weniger lässt sich eine leichte Intelligenzminderung feststellen.

\subsection{Lernstil-Test}

Zur Einschätzung des Lernstils kann man den Fragebogen von Education Planner ${ }^{15}$ benutzen. Er besteht aus 20 Fragen, an dessen Ende die Prävalenz

$\overline{15}$ http://www.educationplanner.org/index.html (10.9. 2018) 
der Lernstile in Prozentzahl wiedergegeben wird. Diese kann dann gedruckt und abgespeichert werden und beinhaltet Tipps für den jeweiligen Lernstil. $\mathrm{Zu}$ unterscheiden ist zwischen dem auditiven, dem visuellen, und dem kinästhetischen Lerntyp. Ein Beispiel kann man in Abbildung 3 sehen:

\section{EDUCATIÖNN PLANNER:}

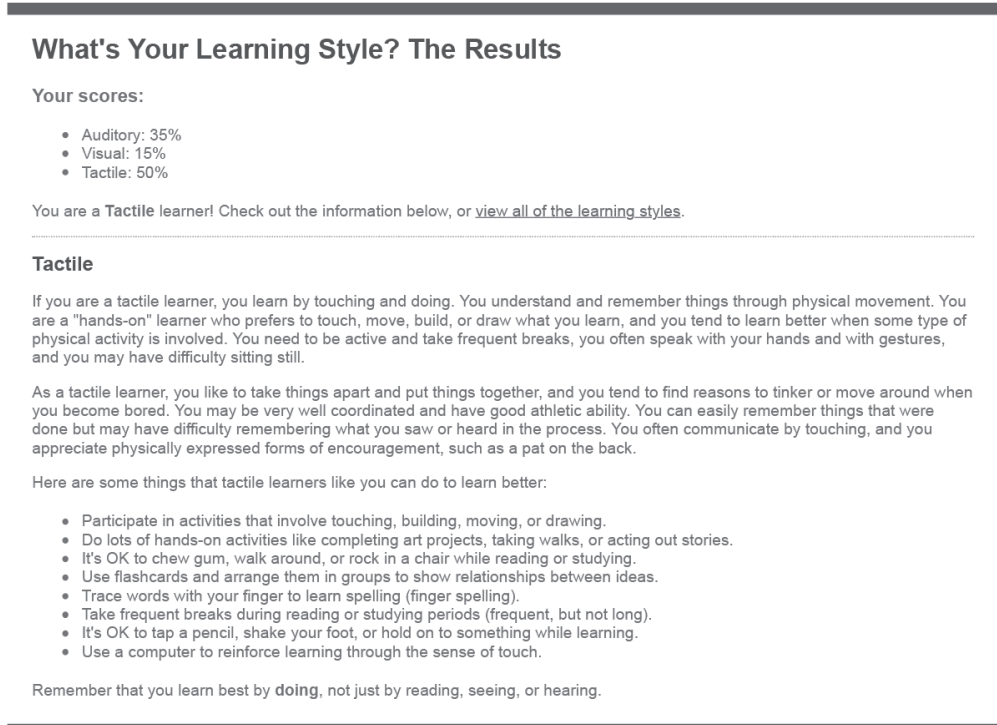

EducationPlanner.org is a public service of the Pennsylvania Higher Education Assistance Agency (PHEAA)
Copyright $(2011$ Pennsylvania Higher Education Assistance Agency All Rights Reserved.

Abbildung 3: Lernstiltest: Testergebnis am Beispiel „kinästhetischer Lerntyp“

\section{FALLSTUDIE}

Während des DaF-Unterrichts mit einem Lernenden bemerkte die Beitragsautorin einige für seine Diagnose untypische Symptome. Sie vermutete eine Intelligenzminderung, von der sie jedoch im Vorhinein nicht informiert wurde. Die Fallstudie hatte das Ziel, einen genaueren Einblick in die Diagnosen des Lernenden zu geben. Es wurden Methoden und Verfahren getestet, welche von einem Lehrer selbst durchgeführt werden können und ihm so bei der Planung einer individuell angepassten Unterrichtsstunde helfen können.

\subsection{Hintergrundinformationen zum Fall "Duma“}

Duma ist ein 17-jähriger Junge. Seine Muttersprache ist Französisch, seine Zweitsprache Schweizerdeutsch; Hochdeutsch lernte er erst in der Schule, jedoch brach er diese nach der zweiten Klasse Grundschule ab. 2016 begann 
er den individuellen Deutschunterricht mit der ersten Autorin dieses Beitrags. Eine Anamnese bekam die Autorin des Beitrags im Voraus nicht zu lesen. Man sagte ihr, er habe ADHS, Dyslexie, Dysgraphie und Dyskalkulie. Die Diagnosen machten auf den ersten Blick auch Sinn: Sie merkte bei dem Probanden den ständigen Bedarf nach Pausen, sein Zappeln, seine unentwickelte, chaotische, mit Rechtschreibfehlern versehene Handschrift. Jedoch untypisch für eine Dyslexie-Diagnose: Er hatte keine Schwierigkeiten mit dem Lautesystem und machte beim Lesen nicht viele Fehler. Das Gelesene aber zu verstehen, etwas Gelesenes nachzuerzählen, es sich zu merken und verschiedene Inhalte zu verbinden, dies bereitete ihm große Schwierigkeiten. Etwa ein Jahr später bekam die Beitragsautorin Dumas Anamnese zu lesen, aus der sie erfuhr, dass Duma ein breites Spektrum an Störungen diagnostiziert worden war, darunter Leichte Intelligenzminderung - eine Diagnose die Dyslexie, Dysgraphie und Dyskalkulie ausschließt. So lauteten seine eigentlichen Diagnosen: Hyperkinetische Störung des Sozialverhaltens, Kombinierte Entwicklungsstörung (des Sprechens und der Sprache, der schulischen Fertigkeiten, kognitiv, sozio-emotional usw.) und weitere, die zum Zwecke dieser Arbeit nicht genannt werden, da sie keine Auswirkung auf den (Fremd-)Spracherwerb haben. Um Dumas Lernschwierigkeiten besser verstehen zu können, wurden Tests durchgeführt, dessen Ergebnisse im nächsten Kapitel vorgestellt werden.

\subsection{Durchführung der Test- und Diagnoseverfahren und ihre Ergebnisse}

Um Dumas Lernfähigkeiten und Sprachkenntnisse besser einschätzen zu können, wurden bei ihm folgende Methoden und Verfahren angewendet: eine ADHS-Diagnosecheckliste (s. Abb. 1 im Kapitel 4.1. bzw. die Tabelle 1 im Kapitel 2.1.), der MoCA-Test (mehr im Kapitel 4.2.), die Einschätzung kommunikativer Fähigkeiten und ein Lernstil-Test (mehr im Kapitel 4.3.). Die Tests wurden nach der Anamese durchgeführt. Auch hatte sich die Lehrerin nach Dumas Interessensfeld erkundigt.

Zuerst wurde ein ADHS-Test durchgeführt (s. Abb. 1, Kapitel 4.1. oder Tabelle 1, Kapitel 2.1.). Duma wurde gemäß ICD-10 eine hyperkinetische Störung des Sozialverhaltens diagnostiziert. Der Test half insofern, dass man durch die Symptomkriterien etwas mehr über diesen Subtyp erfahren konnte. Insgesamt sechs seiner Bezugspersonen, d. h. Erzieher, die derzeit mit Duma arbeiteten, schätzten anhand der ADHS Diagnose-Checkliste seine Diagnose ein, dabei waren die Ergebnisse sehr ähnlich - nur bei einer der Bezugspersonen blieb bei Duma eine „Diagnose“ der hyperkinetischen Störung aus. Diefolgenden Symptome sind bei Duma am stärksten ausgeprägt und sollten bei der Gestaltung einer Unterrichtsstunde möglichst beachtet werden: Für das Kernsymptom Unaufmerksamkeit die Symptomkriterien 1. Beachtet häufig Einzelheiten nicht oder macht Flüchtigkeitsfehler bei den 
Schularbeiten, bei der Arbeit oder bei anderen Tätigkeiten, 2. Hat oft Schwierigkeiten, längere Zeit die Aufmerksamkeit bei Aufgaben oder Spielen aufrechtzuerhalten, 4. Führt häufig Anweisungen anderer nicht vollständig durch und kann Schularbeiten, andere Arbeiten oder Pflichten am Arbeitsplatz nicht zu Ende bringen - nicht aufgrund von oppositionellem Verhalten oder Verständnisschwierigkeiten und 6. Vermeidet häufig, hat eine Abneigung gegen oder beschäftigt sich häufig nur widerwillig mit Aufgaben, die länger andauernde geistige Anstrengungen erfordern - z. B. im Unterricht, Hausaufgaben, für Hyperaktivität die Kriterien 3. Läuft häufig herum oder klettert exzessiv in Situationen, in denen dies unpassend ist bei Jugendlichen oder Erwachsenen kann dies auf ein subjektives Unruhegefühl beschränkt bleiben und 5. Ist häufig "auf Achse" oder handelt oftmals, als wäre er "getrieben" und für Impulsivität die Symptomkriterien 3. Unterbricht und stört andere häufig - platzt z. B. in Gespräche oder in Spiele anderer hinein und 4. Redet häufig übermäßig viel - ohne angemessen auf soziale Beschränkungen zu reagieren.

Als nächstes wurde mit Duma der MoCA-Test (s. Kapitel 4.2.) durchgeführt. Im ersten Teil, „Visuospatial/Executive“, muss der Proband in der ersten Aufgabe eine Zahlen-Buchstabenreihe weiterführen, indem er Buchstaben von A bis E mit Zahlen von 1 bis 5 verbindet. Die Reihe beginnt mit 1-A-2 und soll so weitergeführt werden, dass sie mit dem Buchstaben „E" endet. Die zweite Aufgabe besteht darin, einen Würfel nachzuzeichnen, und die dritte, eine Uhr, die Zehn nach elf zeigt, zu zeichnen, wobei Kontur, Zahlen und Zeiger berücksichtigt werden.

Duma schaffte die erste Aufgabe zuerst nicht, doch dann schaffte er sie mit Hilfe der Lehrerin (s. Abb. 4). Dennoch sollten keine fünf Punkte vergeben werden.

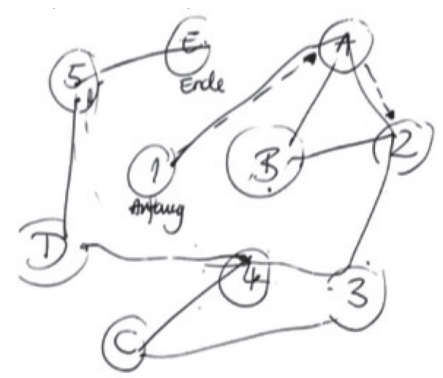

Abbildung 4: Ergebnisse des MoCA-Tests - Zahlen-Buchstabenreihe

Auch die zweite Aufgabe bereitete ihm Schwierigkeiten (s. Abb. 5). Er erzielte keinen Punkt. Die dritte Aufgabe löste er ohne weitere Schwierigkeiten, dennoch wird ein Punkt für die Kontur der Uhr abgezogen. Die Zeiger hat er zwar nicht deutlich groß und klein gezeichnet, erklärte aber „der kleine Zeiger ist auf der Elf, der große auf der Zehn“ (s. Abb. 6). Insgesamt erzielte Duma im ersten Teil zwei Punkte. 


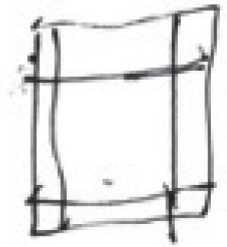

Abbildung 5: Ergebnisse des MoCA-Test - Würfel

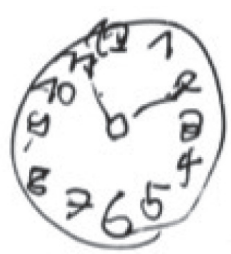

Abbildung 6: Ergebnisse des MoCA-Test - Uhr

Im zweiten Teil, „,Benennen“, muss der Proband drei Tiere benennen - Löwe, Nashorn und Kamel. Drei Punkte werden hier vergeben. Dies löste Duma ohne weitere Probleme und erzielte so drei Punkte.

Der dritte Teil ist eine Gedächtnisaufgabe. Dem Probanden werden fünf Wörter vorgelesen - Gesicht, Samt, Kirche, Tulpe, rot. Der Proband soll die Wortliste in zwei Durchgängen wiederholen. Hier werden derzeit keine Punkte vergeben; es wird im Nachhinein die Erinnerungsfähigkeit des Probanden getestet und bewertet. Duma wiederholte in beiden Durchgängen alle Wörter. Als ihm erklärt wurde, er müsse sich diese Wörter merken und im Nachhinein wiederholen können, sagte er, dass er glaube, dies würde ihm sicher schwerfallen.

Der vierte Teil des Testes widmet sich der Aufmerksamkeit und besteht aus drei Aufgaben. In der ersten Aufgabe wird dem Probanden eine Zahlenliste vorgelesen, die er in der vorgegebenen Reihenfolge wiederholen soll; dann wird eine zweite Zahlenliste vorgelesen, die er rückwärts wiederholen soll. Dafür werden zwei Punkte vergeben. Duma erzielte beide Punkte. In der zweiten Aufgabe wird dem Probanden eine Buchstabenliste vorgelesen, ungefähr ein Buchstabe pro Sekunde, und der Proband soll bei jedem Buchstaben "A $\mathrm{A}^{\text {" }}$ mit der Hand klopfen. Hierfür wird ein Punkt vergeben. Duma hatte großen Spaß an dieser Aufgabe, löste sie fehlerfrei und erzielte den Punkt. Die dritte und letzte Aufgabe zur Aufmerksamkeit macht eine Rechenaufgabe aus. Der Proband muss fortlaufend die Zahl 7 abziehen, beginnend mit 100. Für diese Aufgabe werden drei Punkte vergeben. Da Duma nur die erste Subtraktion richtig löste, erzielt er nur einen Punkt (s. Abb. 7).

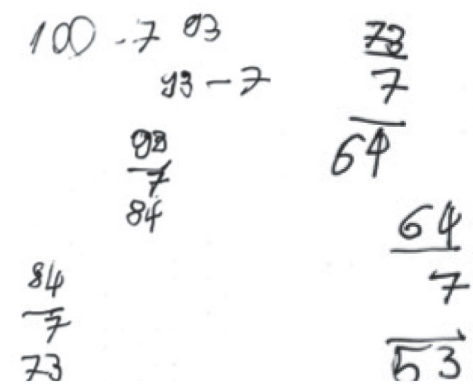

Abbildung 7: Ergebnisse des MoCA-Tests - Fortlaufendes Abziehen 
Der fünfte Teil des Tests widmet sich der Sprache. In der ersten Aufgabe werden dem Probanden zwei Sätze vorgelesen, die er dann wiederholen muss; zwei Punkte werden vergeben. In beiden Sätzen ließ Duma ein Wort aus oder machte eine andere, kleine Veränderung. Dennoch dürfen hierfür keine Punkte vergeben werden. Für die zweite Aufgabe muss der Proband innerhalb einer Minute möglichst viele Wörter nennen, die mit dem Buchstaben „F“ beginnen. Ein Punkt wird vergeben. Duma schrieb nur sieben Wörter und erzielte so keinen Punkt (s. Abb. 8).

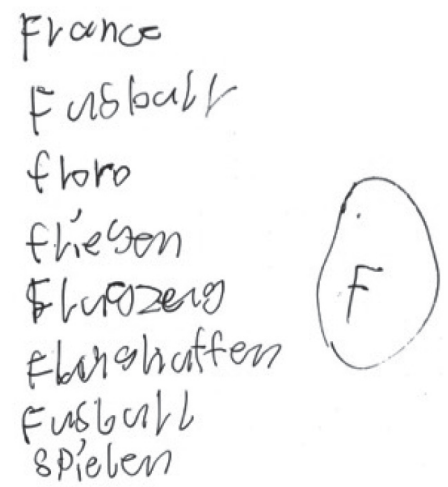

Abbildung 8: Ergebnisse des MoCA-Tests - Wortfeld zum Buchstaben "F"

Im sechsten Teil, "Abstraktion“ muss der Proband eine Gemeinsamkeit von Wortpaaren Eisenbahn-Fahrrad und Uhr-Lineal nennen. Zwei Punkte werden für diese Aufgabe vergeben. Duma nannte die „Verkehrsmittel“ als Gemeinsamkeit von dem Wortpaar Eisenbahn-Fahrrad und "Zahlen" für das Wortpaar Uhr-Lineal und erzielte zwei Punkte.

Dieser Aufgabe folgt die Erinnerungsaufgabe: Der Proband muss sich nun an die Wörter aus dem dritten Teil des Testes erinnern und sie nennen. Man kann dem Probanden auch Hinweise zu Kategorie geben, um ihm zu helfen. Insgesamt fünf Punkte werden vergeben, jedoch nur bei richtigem Nennen ohne Hinweis. Er konnte Gesicht, Kirche, Tulpe und rot reproduzieren, an das Wort "Samt" konnte er sich nicht erinnern. Er erzielte so vier Punkte.

Der achte und letzte Teil des MoCA-Tests widmet sich der Orientierung. Der Proband muss Datum, Monat, Jahr, Wochentag, Ort und Stadt nennen. Hier werden sechs Punkte vergeben. Duma löste diese Aufgabe fehlerfrei und erzielte so sechs Punkte.

Duma erzielte in allen Aufgaben des Moca-Tests 21 Punkte, bzw. 22, da er weniger als 12 Jahre Ausbildung hatte. Da sich für 25 Punkte oder weniger eine leichte Intelligenzminderung feststellen lässt, stimmen auch diese Testergebnisse mit seiner Diagnose überein. Der Test führte $\mathrm{zu}$ einem besseren Verständnis der Diagnose: Im Zusammenhang mit Sprache und Sprachfähigkeit konnte beobachtet werden, dass Duma keine 
Schwierigkeiten mit der Wahrnehmung von Lauten aufzeigt (erkennbar durch die Aufgabe mit Klopfen zum Buchstaben ", $\mathrm{A}$ "), doch große Probleme mit der Rechtschreibung hat (s. Abb. 8 - Wortfeld zum Buchstaben „F“), und dass er, für einen Jungen seines Alters, eine unentwickelte und schlecht lesbare Schrift hat (s. auch Abb. 8 - Wortfeld zum Buchstaben „F“). Er hat wahrscheinlich einen sehr eingeschränkten Wortschatz, was sich auch aus den Ergebnissen der Aufgabe Wortfeld zum Buchstaben „F“ erschließen lässt. Sätze und Texte dürfen nicht zu komplex sein, da er sonst den roten Faden verlieren könnte (z. B. bei der Wiedergabe von Sätzen). In der Unterrichtsplanung sollte man sich also möglichst mit der Einübung von Wortschatz und Rechtschreibung befassen. Vorgegebene Sätze und Texte sollten nicht zu komplex sein, es sollten nicht zu viele neue Wörter eingeführt werden und die Rechtschreibung muss beachtet werden.

Des Weiteren folgte die Einschätzung kommunikativer Kompetenzen, die mittels Muster-Sprachniveautests des ECL (European Consortium for the Certificate of Attainment in Modern Languages) durchgeführt wurden. Duma wurde auf der Stufe B1 getestet. Der ECL-Test (Stufe B1) ${ }^{16}$ besteht aus der mündlichen Prüfung (Hörverstehen und mündliche Kommunikation) und der schriftlichen Prüfung (Leseverstehen und schriftliche Kommunikation). Für jedes Teilgebiet werden 25 Punkte vergeben, so können mit dem gesamten Test 100 Punkte erzielt werden. Die Bewertung verlief mit Berücksichtigung seiner Lernschwierigkeiten; die Durchführung der Einschätzung war zeitlich etwas länger und es wurden bei der Auswertung der schriftlichen Kommunikation mehr Fehler und kürzere Antworten erlaubt. Den mündlichen Teil der B1-Prüfung löste Duma mit 73,5 \%, den schriftlichen mit $47,5 \%$ und bestand so die gesamte ECL-Prüfung für die Stufe B1 gerade noch mit $60,5 \%$.

Zur Einschätzung des Lernstils wurde mit Duma eine Selbsteinschätzung durgeführt. Benutzt wurde der Fragebogen von EducationPlanner (s. Kapitel 4.3.). Den Lernstiltest hat Duma gerne und schnell gelöst und fand die Fragen auch amüsant. Dumas dominanter Lernstil ist der kinästhetische Lernstil. Das bedeutet, dass er „lernt, indem er tut". Über den kinästhetischen Lerntyp, auch haptischer und taktiler genannt, sagt EducationPlanner Folgendes:

Wenn Sie ein haptischer Lerner sind, lernen Sie durch Berühren und Handeln. Sie verstehen und erinnern sich an Dinge durch körperliche Bewegung. Sie sind ein "praktischer" Lerner, der es vorzieht, zu berühren, zu bewegen, zu bauen oder zu zeichnen, was Sie lernen, und Sie neigen dazu, besser zu lernen, wenn eine Art von körperlicher Aktivität involviert ist. Sie müssen aktiv sein und häufige Pausen machen, Sie sprechen oft mit Ihren Händen und mit Gesten, und Sie können Schwierigkeiten haben, still zu sitzen.

$\overline{16}$ https://eclexam.eu/sample-test-german/ (10. 9. 2018) 
Als tastender Lerner nehmen Sie gern Dinge auseinander und setzen sie zusammen und Sie neigen dazu, Gründe zu finden, um herumzubasteln oder herumzulaufen, wenn Ihnen langweilig wird. Sie können sehr gut koordiniert sein und gute athletische Fähigkeiten haben. Sie können sich leicht an Dinge erinnern, die erledigt wurden, haben aber möglicherweise Schwierigkeiten, sich daran zu erinnern, was Sie dabei gesehen oder gehört haben. Sie kommunizieren oft durch Berührung, und Sie schätzen körperlich ausgedrückte Formen der Ermutigung, wie zum Beispiel einen Klaps auf den Rücken. (EducationPlanner, tactile learning style ${ }^{17}$, übersetzt von der ersten Autorin dieses Beitrags)

EducationPlanner bietet auch einige Tipps für kinästhetische Lerner, die jeder Lehrer in seinen Unterricht inkorporieren kann, z. B. die Aktivitäten so gestalten, dass sich die Schüler bewegen und etwas berühren oder malen können; bei der Einübung der Rechtschreibung z. B. Wörter mit dem Finger verfolgen; häufig kurze Pausen machen. Man kann auch versuchen, Aktivitäten auf Computern/Tabletcomputern zu entwickeln.

Zuletzt wurde Duma nach seinen Interessen gefragt und sollte eine Liste erstellen. Er schrieb Folgendes: Musik, Tanzen, Sport. Da dies nur ein beschränktes Interessenfeld ist, wurden noch weitere Inhalte, welche für ihn besonders interessant sein könnten, besprochen und das Interessenfeld erweitert - zu Musik wurden noch Unterkategorien Hip-Hop, Rap, Schlagzeug und Geschichte der Musik aufgeschrieben, zu Tanzen noch Tanzstile, Geschichte des Tanzes und zu Sport noch Kick-box, Fitwerden und Gesunde Ernährung.

\subsection{Eine Deutschstunde mit Duma - vor und nach der Testsdurchführung}

Vor der Durchführung der oben beschriebenen Tests wurde mit Duma eine Unterrichtsstunde zum Thema „Wohngemeinschaft" durchgeführt. Von den Aktivitäten in dieser Unterrichsstunde machte ihm die Galgenmännchen-Aktivität Spaß, doch verging dies, als er über Vor- und Nachteile eines WG-Lebens reden sollte. Das Mindmap fing er an, doch er wurde wegen erneuten Rechtschreibfehlern wütend und wollte dann nicht weitermachen. Die Hörtextaufgaben und die Wortschatzübung löste er ohne weitere Schwierigkeiten. Darauf sollte eine Diskussion über mögliche Lösungsvorschläge für WG-Probleme folgen, doch er hatte zu diesem Zeitpunkt keine Lust mehr, mitzumachen. Alles „war ihm zu viel“ und er „konnte sich nicht mehr konzentrieren“, so musste die Stunde beendet werden.

Nach der Durchführung der ADHS-Diagnosen-Checkliste, des MoCATests, des Lernstiltests und der Einschätzung kommunikativer Fähigkeiten

${ }_{17} \mathrm{http} / / /$ www.educationplanner.org/students/self-assessments/learning-styles-styles.shtml $\quad$ (7. 10 . 2018) 
wurde eine Liste erstellt, wie eine Unterrichtsstunde mit Duma eigentlich aussehen müsste:

- Die ADHS-Symptome beachtend müssen die Aktivitäten des neuen Unterrichtsentwurfs so gestaltet werden, dass Duma Flüchtigkeitsfehler im Nachhinein selbst einsieht, dass Aktivitäten schnell geschaltet werden, klar aufgezählte und veranschaulichte Arbeitsanweisungen vorgegeben sind und kurze Bewegungspausen eingeplant sind, und dass Duma geleitet viel reden kann.

- Die Intelligenzminderung beachtend dürfen vorgegebene Sätze und Texte nicht zu komplex sein, es sollten nicht zu viele neue Wörter eingeführt werden und die Rechtschreibung muss beachtet werden.

- Die Einschätzung kommunikativer Fähigkeiten zeigt, dass Duma besondere Schwierigkeiten bei den Fertigkeiten Leseverstehen und schriftliche Kommunikation aufweist. Er ist auf der Niveaustufe B1 für deutsche Sprache.

- Dumas Lernstil benötigt viel Bewegung, so sollten Aktivitäten so gestaltet werden, dass Duma sich bewegen kann und etwas berühren oder malen kann; zur Einübung der Rechtschreibung können Wörter mit dem Finger verfolgt werden; es sollten häufige, doch kurze Pausen gemacht werden; es können Aktivitäten auf Computer/ Tabletcomputer entwickelt werden.

- Dumas Interessenfeld beruht auf den Kategorien Musik, Tanz und Sport, aus welchen folgende Inhalte für die Unterrichtsplanung entnommen werden können: Hip-Hop, Rap, Schlagzeug, Geschichte der Musik, Tanzstile, Geschichte des Tanzes, Kick-box, Fit werden, Gesunde Ernährung.

Das Thema der individuell angepassten Unterrichtsstunde nach den durchgeführten Tests war "Gesunde Ernährung“. Da Duma an der Galgenmännchen-Aktivität Spaß hatte, wurde diese wiederholt, um das Thema der Stunde anzukündigen. Die erratenen Begriffe „Ernährungspyramide“, „Gesunde Ernährung“, „Nahrungsgruppen“, „sparsam“, „mäßig“ und „reichlich“ sollte er dann versuchen, mit eigenen Worten zu erklären. Darauf folgte eine Aktivität, in der er Lebensmittel in einer Tasche ertasten, erraten und daraufhin benennen sollte. Von dieser Aktivität war er begeistert. Auch das Gruppieren dieser Lebensmittel in Nahrungsgruppen fand er interessant, wobei die Lehrerin ihm doch helfen musste, die Nahrungsgruppen zu benennen. Es folgte eine kleine Bewegungspause, über die er sich freute. Als Nächstes wurde eine eigene Ernährungspyramide erstellt, indem die Nahrungsmittel in drei Gruppen nach Farben aufgeteilt wurden - der untere grüne Bereich für Lebensmittel, von welchen wir reichlich essen sollten, der mittlere gelbe für jene, von welchen wir mäßig essen sollten, und der obere 
rote für jene, von welchen wir nur sparsam essen sollten. Hier die Abbildung zu diesen Aktivitäten:
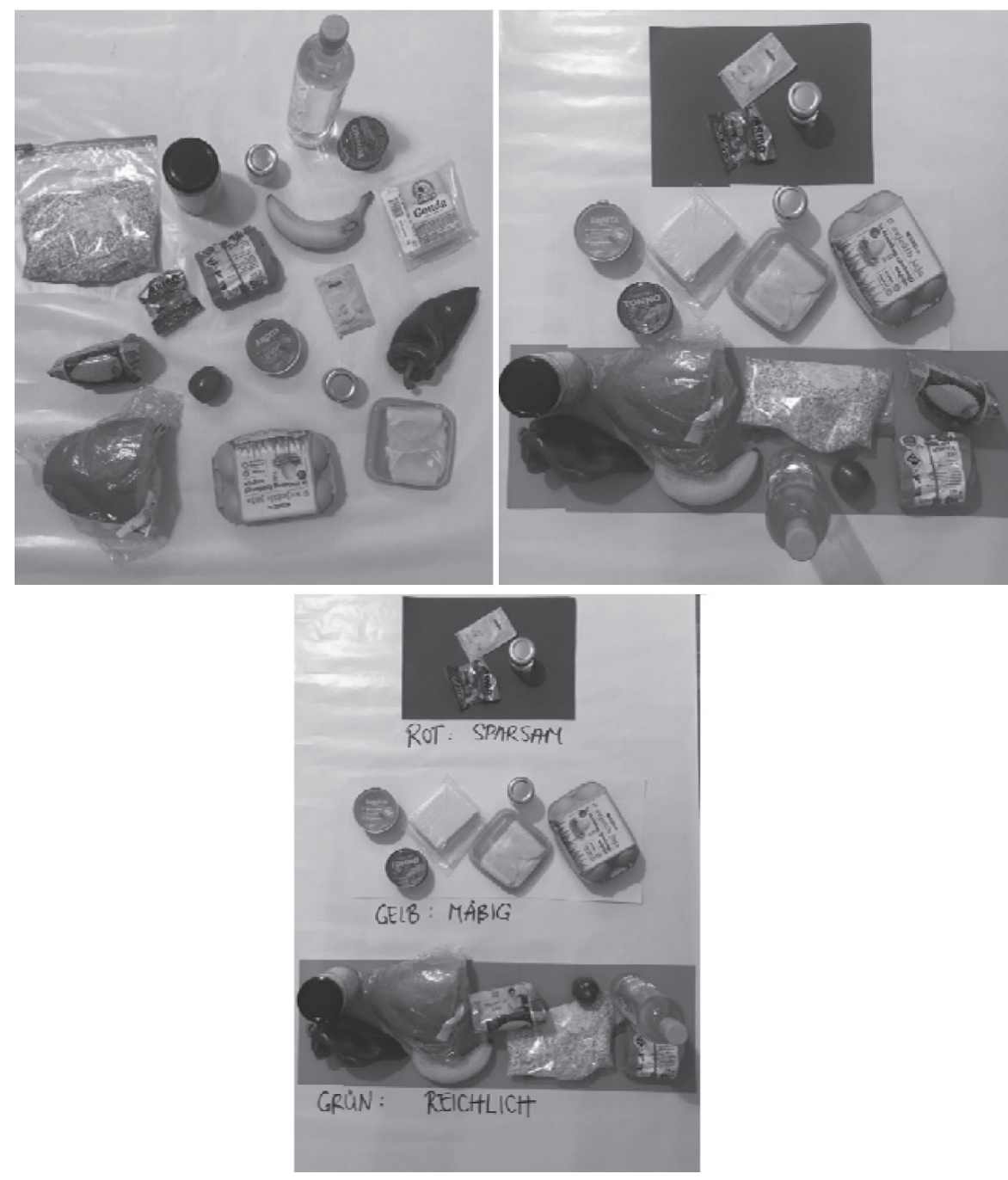

Abbildung 9: Ernährungspyramide

Dann schaute Duma sich ein Video auf YouTube an, damit er seine Ernährungspyramide kontrollieren konnte. Für die Einübung der Rechtschreibung der Begriffe "Ernährungpyramide", "Gesunde Ernährung", „Nahrungsgruppen“, „sparsam“, "mäßig“ und „reichlich" wurde eine Mal-App gewählt, sodass er die Wörter mit dem Finger, anstatt einem Stift schrieb. Dies machte ihm auch viel mehr Spaß, als die Wörter einfach nur ins Vokabelheft zu schreiben. Es folgte nochmals eine kurze Bewegungspause. Danach folgte die Aktivität, in der Duma nacherzählen 
sollte, was er in der Stunde gelernt hatte und dabei sollte er die neuerlernten Begriffe benutzen. Dies verlief ebenso erfolgreich. Zum Schluss sah er sich auf YouTube noch ein Video an. Das Video zeigte ein Selbstexperiment eines jungen Mannes, der versucht, eine Woche keinen Zucker zu essen. Er zählt einige Lebensmittelprodukte auf, in denen überraschenderweise sehr viel Zucker enthalten ist. Duma ging danach in die Lehrerküche und suchte nach Produktpackungen, um dort den Zuckergehalt abzulesen. Dies war die letzte Aktivität der Stunde. Die Lehrerin erklärte Duma noch die Hausaufgabe - er sollte auch zu Hause in den Kühlschrank schauen und aufschreiben, welche Produkte, die er oft zu sich nimmt, Zucker enthalten. Dies war eine der wenigen Hausaufgaben, die er selbstständig ohne Druck erledigt hatte.

Die Unterrichtsstunde war ein Erfolg - alle Aktivitäten wurden durchgeführt, Duma hatte viel Spaß am Lernen, machte seine Hausaufgaben und erzählte von der Stunde noch einige Wochen später. Die angewendeten Verfahren (die ADHS-Diagnosecheckliste, der MoCA-Test, die Einschätzung kommunikativer Fähigkeiten, der Lernstil-Test, die Erkundigung nach dem Interessensfeld) halfen der Lehrerin, d. h. der Beitragsautorin, Duma und seine Lernschwierigkeiten besser zu verstehen. Es war jetzt auch klar, warum frühere Unterrichtsstunden scheiterten und was anders hätte gemacht werden sollen. Die im Nachhinein entworfene Unterrichtsstunde wurde so konzipiert, dass Duma sich viel bewegen und viel tasten konnte. Dies wurde durch Bewegungspausen und durch Ertasten von Realien erreicht. Dank dieser Methoden war Duma während der Lernzeit viel ruhiger und konzentrierter, als er es in der vorherigen Stunde gewesen war, und hatte weniger gezappelt. Auch wurden die Aktivitäten schnell geschaltet, so wurde die eingeschränkte Konzentrationspanne immer wieder "zurückgesetzt”. Den für die Unterrichtsstunde ausgewählten Input machten die YouTube Videos aus, welche den Lernprozess sowohl auditiv als auch visuell unterstützen. Dies ist eine passende Alternative für das Bearbeiten von Lesetexten, was für Duma sehr anstrengend ist - einerseits infolge seiner ADHS, andererseits wegen seiner Intelligenzminderung. Dies wurde auch bei der Einschätzung kommunikativer Fähigkeiten offensichtlich, bei welcher er besondere Schwierigkeiten bei den Fertigkeiten Leseverstehen und schriftliche Kommunikation aufwies.

Aufgrund der ADHS und der Intelligenzminderung ist zu beachten, dass der Lerner nicht mit zu vielen neuen Wörtern überfordert wird - dies ist auch in Bezug auf vorherige Misserfolgserfahrungen sehr wichtig. Es sollten lieber niedrigere, aber reale Lernziele gesetzt werden, sodass der Lerner die Möglichkeit hat, Misserfolge mit Erfolgen zu ersetzen. So wählte die Beitragsautorin für die Unterrichtsstunde sechs Hauptstichwörter, davon fünf unbekannte. Zum Erfassen dieser diskutierten die Beitragsautorin und Duma zunächst gemeinsam und Duma versuchte sie mit eigenen Worten zu 
beschreiben. Die Aktivität "Jetzt bist du der Lehrer" diente nochmals dazu, den erlernten Stoff zu wiederholen, und zwar so, wie es Duma am liebsten tut - indem er viel redet. Aufgrund seiner Lese- und Rechtschreibstörung hat Duma große Probleme und eine große Abneigung gegenüber dem Schreiben. Dennoch muss an seiner Rechtschreibung gearbeitet werden. Die Galgenmännchen-Aktivität nutzte die Beitragsautorin hier als initiales visuelles "Abspeicherbild" für neue Wörter. Anstatt ihn dann mit dem Abschreiben von Vokabeln zu "quälen", benutzte sie hier eine MalApp, mithilfe welcher er die neuen Wörter mit dem Finger schreiben konnte. So wurde nochmals sein taktiler Lernstil beachtet. Das Nutzen des Tabletcomputers ist an sich schon eine Beachtung des Lernstils, da er etwas in der Hand hat. Dabei verbindet Duma im Gegensatz zum Stift mit Technologie keine Misserfolge, sondern Spaß.

Die Erkundigung nach seinem Interessensfeld sorgte ebenfalls für ein geringeres Lern-Zwangsgefühl. Ein Beweis dafür ist sein darauffolgendes Engagement bei den Hausaufgaben und seine Begeisterung für das Thema. Er erzählte noch einige Wochen später davon, wie schlecht Zucker auf den Körper wirkt und insistierte beim wöchentlichen Einkauf auf gesunde Produkte. So hatte er eigentlich selbstständig den nächsten Lernschritt gemacht - er hat die Theorie in die Praxis übertragen und so auch ein Erfolgsgefühl zu der Unterrichtsstunde erlebt.

\section{ALLGEMEINE LEHR- UND LERNTIPPS FÜR DEN UNTERRICHT MIT KINDERN MIT LERNSCHWIERIGKEITEN}

Für eine Unterrichtsstunde mit einem Kind mit Lernschwierigkeiten ist ein erheblich größeres Engagement erforderlich als für ein Kind ohne größere Lernschwierigkeiten. Die Methoden, die täglich benutzt werden, müssen den individuellen Bedürfnissen des Lerners angepasst werden, um einen maximalen Erfolg zu erreichen bzw. um weitere Misserfolgserfahrungen bei dem Lerner zu vermeiden. Im Fall Dumas halfen die präsentierten Verfahren dabei, Duma und seine Lernschwierigkeiten besser zu verstehen. Die Deutschunterrichtsstunde nach den durchgenommenen Testmethoden und Verfahren war, im Gegensatz zu jener davor, ein Erfolg. Dies ist Beweis dafür, dass jeder Lernende anders ist, besonders jene mit mehreren komorbiden Lernschwierigkeiten und dass deren individuelle und spezifische Bedürfnisse aus keiner Diagnoseliste entnommen werden können. Es ist für den Lernenden und für seinen erfolgreichen Spracherwerb von größter Wichtigkeit, dass sich die Lehrperson mit ihm und seinen Bedürfnissen bekannt macht, um die beste Herangehensweise zu entwickeln, Misserfolgserfahrungen zu vermeiden und maximalen Erfolg erreichen zu können. 
Im Folgenden werden, einige Ratschläge präsentiert, die bei der Vorbereitung einer individuell angepassten (Fremdsprachen-) Unterrichtsstunde und beim Lernen mit einem Kind mit Lernschwierigkeiten von Nutzen sein können:

1. Regelmäßiges Wiederholen (Born und Oehler, 2005): Leidet der Lerner an einer reduzierten Aufmerksamkeitsspanne oder einer begrenzten Aufnahmekapazität, sollte erlernter Stoff regelmäßig wiederholt werden. Dies kann entweder durch Hausaufgaben erreicht werden, oder man verwendet einige Minuten der darauffolgenden Unterrichtsstunde für eine Wiederholungsübung. So können Lerninhalte aus dem Kurzzeit- in das Langzeitgedächtnis übergehen. Besonders geeignet sind z. B. auch Karteikarten, die in einer Karteikartenbox in zwei Spalten geordnet sind - eine Spalte für Inhalte, die erlernt sind, die andere für Lerninhalte, die weiterhin wiederholt und geübt werden sollen.

2. Wenige, doch erfolgreiche Methoden (ebd.): Während in der alltäglichen Unterrichtssituation viele unterschiedliche Methoden das Interesse der Lernenden wecken, bedeuten diese bei Kindern mit Lernschwierigkeiten, bei welchen meistens selbst die Konzentration und Motivation eine Herausforderung sind, nur eine weitere Unsicherheit. Stattdessen sollten neue Methoden nur hier und da ausprobiert werden; Methoden, die sich als erfolgreich erweisen, sollten auch weiterhin angewandt werden, denn Kinder mit Lernschwierigkeiten wissen gerne im Voraus, was von ihnen erwartet wird. Mit der Zeit beherrschen die Lernenden eine Methode und können dann den Stoff auch schneller aufnehmen.

3. Reduzierte, doch realistische Ziele (ebd.): Nicht alle Lerner sind gleich und nicht jeder Lerner kann einen Stoff, so einfach er auch scheint, erwerben. Lernziele sollten so auf das Wichtigste reduziert werden, um einerseits den Lernenden nicht $\mathrm{zu}$ überfordern, andererseits um Erfolgserfahrungen zu ermöglichen. Das Kind sollte für jedes erreichte Ziel gelobt werden - damit werden Erfolgserfahrungen und Motivation geschaffen, sowie eine zukünftige Lernabneigung verringert. Mit der Zeit können dann Lernziele erweitert und erhöht werden.

4. Erfolgserfahrungen ermöglichen(ebd.): Wieschonerwähnt, leiden die meisten Kinder mit Lernschwierigkeiten an Misserfolgserfahrungen. Ständige Ermutigung ist bei solchen Kindern von äußerster Wichtigkeit. Hat ein Kind die Einstellung „Ich kann das nicht" und „Hat ja eh keinen Sinn", wird es durch weitere Misserfolge nur noch mehr Abneigung gegenüber dem Lernen entwickeln. Man sollte 
solche Kinder immer wieder für ihre Erfolge loben, um so auch ihr Selbstwertgefühl zu pflegen.

5. Anknüpfung an das Interessensfeld: Wird der Lernstoff dem Interessensfeld des Lernenden angeknüpft, wird dieser schon im Voraus einen geringeren Lern-Zwang bzw. eine geringere Lernabneigung aufweisen. Leidet ein Kind z. B. unter einer Lesestörung, weist aber Interesse für Pferde, Musik, Computer oder andere beliebige Themen auf, können ihm Lesetexte geboten werden, aus denen er etwas Neues zu seinem Interessensfeld lernt. Dabei sollte man jedoch die Länge und die Komplexität seiner Lernschwierigkeit anpassen.

6. Angepasster Input (ebd.): Es ist äußerst wichtig, den Input dem Kind mit Lernschwierigkeiten anzupassen. Leidet ein Kind an einem Aufmerksamkeitsdefizit oder einer Intelligenzminderung, sollten z. B. Hör- und Lesetexte nicht komplex sein, visueller Input nicht abstrakt sein und die Sprache des Inputs sollte auf der entsprechenden Sprachniveaustufe beruhen, denn solche Kinder werden nur selten etwas "aus dem Kontext heraus" verstehen. Leidet ein Kind unter einer Lese- und Rechtschreibstörung, sollte man es möglichst wenig mit Lesetexten konfrontieren. Müssen diese dennoch benutzt werden, kann man dem Lerner helfen, indem man wichtige Stichwörter unterstreicht und dem Lerner so einen Leitfaden bietet.

7. Pausen (ebd.): Für Kinder mit ADHS sind Pausen während des Lernprozesses besonders wichtig. Handelt es sich um einen "Schwärmer", kann man ihm kurze Ruhepausen gönnen; "Zappelmänner" profitieren vielmehr von einer Bewegungspause. Die Unterrichtsstunde sollte möglichst in Intervalle von bis zu 15 Minuten unterteilt werden, nach welchen eine Pause folgt. Die Pause dient als eine Erholung vom Lernen oder als ein "Reset" der Aufmerksamkeit. Mit der Zeit können die Intervalle verlängert werden. In jedem Fall wirken die Pausen motivierend auf die Kinder, denn sie wissen schon im Voraus, dass sie nicht überfordert werden.

8. Geduld und Unterstützung (ebd.): Sei es von den Eltern oder von der Lehrkraft, Kinder mit Lernschwierigkeiten sind auf viel Unterstützung angewiesen. Von einem Kind mit Lernschwierigkeiten kann nicht erwartet werden, dass es die Hausaufgaben, zumindest im frühen Lebensalter, völlig selbständig löst. Hier wird von allen Teilnehmern des Lernprozesses viel Geduld erwartet. Keinesfalls darf das Kind die Verzweiflung der Lehrkraft oder der Eltern spüren. Diese Verzweiflung "spiegeln“ die Kinder und verlieren an Motivation, denn sie denken: „sehen meine Eltern/Lehrer darin 
keinen Sinn, hat es sicher auch keinen Sinn" und es werden weitere Misserfolgserfahrungen angereiht. Stattdessen sollten Eltern, die sich mit dieser Aufgabe überfordert fühlen, Hilfe von einer erfahrenen Lehrkraft in Form von Nachhilfe aufsuchen.

9. Beachtung des Lernstils: Oft verlieren Lernende Motivation und Interesse, weil ihre Lernstile nicht mit dem Lehrstil des Lehrers übereinstimmen. So wird sich ein visueller Lerntyp langweilen, wenn die Unterrichtsstunde einzig und allein auf Hörtexten und auditivem Input beruht. Kinder mit einer hyperkinetischen Störung lernen so z. B. viel erfolgreicher, wenn sie während der Unterrichtsstunde viel tasten, malen und sich bewegen können. Hier erweist sich die Nutzung von Medien wie Computern oder Tabletcomputern als besonders hilfreich. Die Einschätzung des Lernstils benötigt wenig Aufwand, kann jedoch einen großen Unterschied in der Lernsituation ausmachen.

10. Nonverbale Fehlerkommunikation (ebd.): Für Kinder mit Lernschwierigkeiten ist das Motto "aus Fehlern wird gelernt" problematisch, da sie sich meist $\mathrm{zu}$ oft mit zu vielen Fehlern auseinandersetzen müssen. Wird ständig nur auf Fehler aufmerksam gemacht, reagieren sie selten mit der Einstellung „Super, kann ich verbessern", sondern meist mit "Schon wieder", „Immer mache ich alles falsch", und "Kann ich eh nicht, ist mir egal". Dabei werden manche Kinder sogar wütend und aggressiv, wenn durch verbale Ausdrücke wie „Das ist ein Fehler" oder „Das ist falsch“ auf Fehler aufmerksam gemacht wird, da diese an sich schon eine negative Konnotation haben. Stattdessen kann man versuchen, nonverbale Fehlerkommunikation anzuwenden. Macht ein Kind mit Lese- und Rechtschreibstörung z. B. einen Fehler in der Rechtschreibung oder beim Lesen eines Wortes/Satzes, kann man ihn z. B. fest drücken/ umarmen, ein wenig kitzeln oder bei der Hand nehmen; eben einen nonverbalen Ausdruck benutzen, welchen der Lernende mit einer positiven Konnotation erlebt. Der Lernende muss sich jedoch darüber im Klaren sein, was dieser Ausdruck bedeutet.

11. Mindmaps (Born und Oehler, 2005; Galić-Jurušić, 2004): Bei Kindern mit ADHS, Intelligenzminderung und Lese-Rechtschreibstörung zeigen sich Mindmaps als eine besonders geeignete Lern- und Abspeicherungsmethode. Die Assoziogramme/Gedächtniskarten bieten einen vereinfachten Überblick des Stoffes - es werden nur die wichtigsten Stichwörter veranschaulicht und es muss bei der Vorbereitung nur wenig geschrieben, und beim Wiederholen nur wenig gelesen werden. Die Mind-Maps bieten die Möglichkeit einer einfachen und sinnvollen Strukturierung und können auf 
verschiedenste Art und Weise erstellt werden; schriftlich auf einem Blatt Papier, auf einem Computer oder Tabletcomputer, durch Techniken wie Malen, Zeichnen, Collage. Auch sind sie für fast alle Lernfächer geeignet.

Das Beachten individueller Lernschwierigkeiten der Schüler wie auch die Anwendung dieser Ratschläge sorgt für ein vermindertes Zwangsgefühl beim Lernen, schafft Erfolgserfahrungen und Motivation und bringt mehr Freude und Fröhlichkeit in den Unterricht mit Kindern mit Lernschwierigkeiten.

\section{LITERATUR}

Born, A. und Oehler C. (2005) Lernen mit ADS-Kindern. Ein Praxishandbuch für Eltern, Lehrer und Therapeuten. 4., aktualisierte Auflage. Stuttgart: W. Kohlhammer GmbH.

Döpfner, M., Görtz-Dorten, A. und Lehmkuhl, G. (2008) Diagnostik-System für Psychische Störungen im Kindes- und Jugendalter nach ICD-10 und DSM-IV, DISYPS-II. Bern: Huber.

Döpfner, M., Fröhlich J. und Lehmkuhl G. (2013) Aufmerksamkeitsdefizit-/Hyperaktivitätsstörung (ADHS). Göttingen: Hogrefe Verlag.

Galić-Jurušić, I. (2004) Djeca s teškoćama u učenju: rad na spoznajnom razvoju, vještinama učenja, emocijama i motivaciji. Lekenik: Ostvarenje.

Gawrilow, C. (2016) Lehrbuch ADHS: Modelle, Ursachen, Diagnose, Therapie. 2. Auflage. München: Reinhardt Ernst Verlag.

Gold, A. (2011) Lernschwierigkeiten: Ursachen, Diagnostik, Intervention. 1. Auflage. Stuttgart: W. Kohlhammer $\mathrm{GmbH}$.

Holowenko, H. (1999) Das Aufmerksamkeits-Defizit-Syndrom (ADS). Weinheim und Basel: Beltz Verlag.

James, W. (1890) Principles of Psychology (Vol I and II). New York: Henry Holt and Company.

Miles, T .R. und Miles, E. (1999) Dyslexia: A Hundred Years On (2 $2^{\text {nd }}$ edition). Buckingham: Open University Press.

Tischler, L. (2017) Das doppelte Diskrepanzkriterium. VIGESCO: Institut für psychologisch-pädagogische Bildung und Entwicklung. https://vigesco-institut.de/blog/das-doppelte-diskrepanzkriterium (11.9. 2018)

Walter, J. und Wember F. B (Hrsg.) (2007) Sonderpädagogik des Lernens. Göttingen: Hogrefe Verlag.

Wendlandt, W. (2006) Sprachstörungen im Kindesalter: Materialien zur Früherkennung und Beratung. Stuttgart und New York: Georg Thieme Verlag. 


\section{USVAJANJE STRANOGA JEZIKA U DJECE S POTEŠKOĆAMA U UČENJU: STUDIJA SLUČAJA}

U ovom su radu predstavljeni rezultati istraživanja jednoga slučaja s ciljem pronalaženja odgovarajućeg načina rada s djecom koja imaju poteškoće u učenju, gdje je fokus na usvajanju stranoga jezika. Glavno je težište rada na pronalasku odnosno definiranju poteškoća u učenju, tipova poremećaja i uzroka koji otežavaju učenje. U radu se detaljno pojašnjavaju različite poteškoće u učenju kod učenika kao što su ADHD, disleksija, smanjena inteligencija i poremećaj u razvoju jezika, s naglaskom na ADHD i učenje njemačkog jezika na razini B1. Istraživanje se bavi slučajem Duma, dječakom s više komorbidnih poteškoća u učenju, a u njemu su detaljno opisane metode i postupci koji bi mogli pomoći u planiranju individualno prilagođenih nastavnih sati s učenicima s poteškoćama, primjerice dijagnostički test za ADHD, MoCA test, procjena komunikacijskih vještina i test stila učenja koje svaki nastavnik može sam provesti.

Ključne riječi: usvajanje jezika, poteškoće u učenju, ADHD, disleksija, poremećaj u razvoju jezika, mentalna retardacija, studija slučaja.

\section{FOREIGN LANGUAGE ACQUISTION IN CHILDREN WITH LEARNING DIFFICULTIES: A CASE STUDY}

The present paper describes the results of a case study in the context of language acquisition in children with learning difficulties. The paper focuses on learning difficulties, more specifically, on disorders and causes that interfere with learning German as a foreign language, such as ADHD, dyslexia, mental retardation and language developmental disorder. The emphasis is put on ADHD and teaching German as a foreign language at the B1 level. The presented case study is the case of Duma, a boy with several co-morbid learning difficulties. There are methods and procedures described that have been used to help plan a personalized lesson - an ADHD diagnostic checklist, the MoCA test, assessment of communicative skills, and a learning style test, which can be conducted by every language teacher.

Keywords: language acquisition, learning difficulties, ADHD, dyslexia, developmental language disorder, mental retardation, case study. 